\title{
Virer et tourner attributifs: De l'analyse quantitative des cooccurrences aux contrastes sémantiques
}

\author{
PETER LAUWERS AND NIEK VAN WETTERE \\ Université de Gand \\ peter.lauwers@ugent.be; niek.vanwettere@ugent.be
}

\begin{abstract}
Résumé
La présente contribution porte sur tourner et virer, deux verbes attributifs (p. ex. elle a virél tourné folle) qui ne sont pas encore reconnus pleinement par la tradition grammaticale normative ni dans les travaux des linguistes. Elle vise à établir le profil distributionnel / collocationnel de ces deux verbes qui semblent opérer dans le même domaine sémantico-fonctionnel, afin de mettre à nu les subtiles divergences au niveau de leur profil sémantique. Celles-ci sont rattachées à la sémantique de base du verbe lexical, laissant entrevoir dans ces emplois grammaticalisés des effets dus à la «persistance lexicale » (lexical persistence). Afin d'objectiver au maximum les profils distributionnels des deux verbes (et partant leur profil sémantique), les outils statistiques offerts par la méthode collostructionnelle (Stefanowitsch et Gries 2003; Gries et Stefanowitsch 2004) sont mis à profit. En outre, la combinaison de l'approche collocationnelle et de l'analyse morphosyntaxique amène à distinguer l'emploi proprement attributif des deux verbes d'un emploi directionnel abstrait homonymique (l'entreprise a viré solaire), qui est encore proche de la construction prépositionnelle (virer à / tourner à).
\end{abstract}

Mots clés: verbe attributif, quasi-synonymie, analyse collexémique, spécialisation sémantique, directionnel

\begin{abstract}
This article deals with tourner and virer, two attributive verbs (e.g., elle a viré/tourné folle 'she went crazy') that have not yet been fully recognized by prescriptive grammar or in linguistic work. We aim to establish the distributional/collocational profile of these two verbs, which seem to operate in the same semantic-functional domain, in order to reveal the subtle differences in their semantic properties. These differences have to do with the basic semantics of the lexical verb, and allow one to discern, in grammaticalized uses, effects due to lexical persistence. In order to make the distributional properties of the two verbs as objective as possible, (and thus their semantic properties), the statistical tools provided by the collostructional
\end{abstract}

Nous tenons à remercier nos trois relecteurs anonymes. Leurs remarques nous ont permis de clarifier considérablement notre propos. 
method (Stefanowitsch et Gries 2003; Gries and Stefanowitsch 2004) are used to advantage. In addition, the combination of the collocational approach and morphosyntactic analysis leads us to distinguish between the properly attributive use of the two verbs, and a homonymic abstract directional use (l'entreprise a viré solaire 'the business went solar'), which remains close to the prepositional construction (virer à / tourner à).

Keywords: attributive verb, quasi-synonymy, collexemic analysis, semantic specialization, directional

\section{INTRODUCTION : DEUX MAL-AIMÉS DE LA GRAMMAIRE FRANÇAISE}

Cet article est consacré à virer et tourner, deux verbes attributifs relativement récents (tourner : ${ }^{\circ} 1862$; virer : ${ }^{\circ} 1926$; cf. Van Wettere et Lauwers 2017), qui n'ont pas encore été reconnus pleinement dans les ouvrages de référence ${ }^{1}$.

(1) l'on s'en va avec ça si les bons tournent méchants!

(Sketch Engine)

(2) Tous les vilains de la ville la détestent, évidement [sic], mais surtout Poison Ivy, qui, avant qu'elle ne vire méchante, avait étée [sic] son amie

(Sketch Engine)

Cette contribution s'inscrit donc dans une série d'études qui ont montré que le nombre de verbes attributifs (être, devenir, rester, faire (vieux), passer (médecin), etc.) est plus grand qu'on ne le pense (Leeman 1996, Lamiroy et Melis 2005, Lauwers et Tobback 2010). Le statut attributif peut tout d'abord être déduit de la nature morphosyntaxique du complément du verbe. Celui-ci est de nature adjectivale et s'accorde en genre et en nombre avec le sujet (ex. (1-2)), ou prend la forme d'un nom nu (c.-à-d. sans article, p. ex. l'atmosphère avait viré ghetto, Sketch Engine). Le constituant attributif est syntaxiquement obligatoire et exprime une caractéristique du sujet. C'est lui qui fonctionne comme principal sélectionneur du sujet, le verbe étant peu sélectif (v. Lamiroy et Melis 2005). Si les verbes attributifs prototypiques (les copules, p. ex. être, devenir) acceptent la pronominalisation de l'attribut en le (invariable), force est de constater que virer et tourner ne s'y prêtent pas (Méchant? *Il l'a tourné, en effet).

Sur le plan du sens, virer et tourner expriment un changement d'état, ce qui les rattache au paradigme des verbes devenir, se faire, passer (chef), etc. (Lauwers et Tobback 2010). Les exemples (1) et (2) montrent déjà qu'ils peuvent facilement passer pour des (quasi-)synonymes, ce qui est confirmé par les définitions fournies par le Petit Robert (2010) : 'changer, évoluer pour devenir' (pour tourner attributif), 'devenir' (pour virer attributif).

Si les constructions attributives de tourner et de virer sont déjà recensées par le Petit Robert (2010), il n'en est pas de même du Trésor de la Langue française (informatisé) (TLFi) et du Grand Larousse (1971-1978), ouvrages plus anciens, qui ne reconnaissent pas encore $<$ tourner + attribut $>$. En outre, force est de constater que tourner et virer attributifs passent à travers les mailles du filet de bon nombre de

\footnotetext{
${ }^{1}$ Abréviations : ADJ : adjectif; AO : attribut de l'objet; COD : complément d'objet direct; fq : fréquence; $\mathrm{N}$ : nom; SN : syntagme nominal; SPrép : syntagme prépositionnel; s.v. : à l'article.
} 
grammaires de référence et même de dictionnaires de constructions verbales (v. Lauwers et Tobback 2010 pour un aperçu). Même les publications spécialisées (Leeman 1996, Lamiroy et Melis 2005) ne les relèvent pas encore. Le registre familier, voire populaire (virer : «fr. fam. à la mode en France », Grevisse et Goosse 2007 : 263; « le populaire virer», Wilmet 2007 : 517), auquel on les confine, et parfois aussi leur caractère régional (tourner : « usages régionaux ou populaires », Grevisse et Goosse 2007 : 263; v. aussi Wilmet 2007 : 517), y sont pour beaucoup. Un autre frein à leur reconnaissance officielle est la concurrence avec la construction indirecte en $\grave{a}$, qui, plus ancienne, semble beaucoup mieux entérinée (Leeman 1996, Petit Robert 2010, TLFi, Grand Robert 2016, entre autres). Enfin, pour tourner, il s'y ajoute un problème de délimitation par rapport aux compléments adverbiaux réalisés par des adjectifs invariables (tourner rond/sec/court), ce qui crée parfois un problème d'accord (accord ou absence d'accord avec le sujet?) et partant des stratégies d'évitement. Ce problème se manifeste notamment dans la vis tourne folle, phrase que l'on « entend pourtant » (Wilmet 2007 : 517). Il n'est donc pas surprenant que ces deux verbes attributifs reproduisent le schéma classique de l'ascension sociale de mots anciennement confinés au français 'non conventionnel', qui passe nécessairement par des réactions critiques de la part des locuteurs soucieux de la stabilité de la norme :

«Quelqu'un ici saurait-il d'où sort cette expression « virer en tête » entendue à satiété hier soir (élections) : machin vire en tête à Machouilly-les-oies, trucmuche vire en tête à Gargoulin-les patates, etc. C'est manifestement le nouveau tic langagier des bavards télévisuels (pas au point de supplanter « et bien » évidemment) : où ont-ils attrapé ça? » $<$ http://correcteurs.blog.lemonde.fr/2014/03/22/le-telesiege-enseignement/> consulté le $15 / 05 / 2016$

Toutefois, grâce aux grands corpus, nous sommes désormais en mesure d'examiner ce genre de questions en dehors de toute préoccupation prescriptive. En fait, pour l'instant, seuls les corpus web s'avèrent suffisamment grands pour mener des analyses quantitatives de phénomènes d'innovation lexicale, ou plus précisément de lexique-grammaire, observés dans l'usage. Ces corpus web s'imposaient d'autant plus ici que le phénomène lexical reste fortement lié à la langue familière et qu'il fallait donc un corpus dans lequel ce registre soit suffisamment représenté. Nous partons du principe qu'ils nous permettront de répondre aux questions suivantes :

(i) Si la reconnaissance de ces deux verbes attributifs s'impose désormais, il reste à faire la description de leur profil distributionnel et, plus spécifiquement, collocationnel. Plus précisément : avec quels attributs et, dans une moindre mesure, avec quels sujets (ces derniers étant en grande partie sélectionnés par l'attribut) apparaissent-ils? Certaines combinaisons tendent-elles au figement?

(ii) L'analyse de ces profils distributionnels au moyen de techniques statistiques nous permettra de préciser et d'objectiver le sens de ces deux verbes attributifs et notamment de mettre en évidence ce qui distingue ces deux quasi-synonymes. Ensuite, il conviendra de rattacher ces différences au profil sémantique global des deux verbes, à la recherche de traces de la «persistance lexicale » (lexical persistence, Hopper 1991) dans les emplois attributifs, qui sont en effet des emplois grammaticalisés. 
(iii) Au-delà de la dimension lexicale, les efforts conjugués de l'analyse morphosyntaxique et de l'analyse collocationnelle feront apparaitre deux constructions homonymes, identiques en surface, mais différentes du point de vue syntaxique et sémantique.

Le corpus retenu pour notre étude est le French Ten Ten Corpus, version 2.0. [2012] (Kilgarriff et al. 2014), qui fait partie de la famille de corpus web étiquetés interrogeables par le biais de l'interface Sketch Engine. Il s'agit d'un corpus 'tout fait' qui a été compilé à l'aide de SpiderLing (web crawler), nettoyé et étiqueté à l'aide de Tree Tagger. Comme tout corpus web de ce type, sa composition est assez hétérogène, comportant une grande variété de données web (textes journalistiques, Wikipédia, forums, etc.). Le nombre total de mots s'élève à presque dix milliards.

Dans ce corpus, nous avons relevé toutes les occurrences des deux verbes (forme lemmatisée) suivis directement d'un adjectif ou d'un nom. L'analyse a été exhaustive, sauf pour <tourner + nom>, combinaison pour laquelle nous avons extrait des 38826 attestations initiales un échantillon randomisé de 6647 occurrences à l'aide de la fonctionnalité prévue à cet effet par l'interface du corpus. Ce nombre correspondait au nombre d'occurrences de <virer + nom $>$. Cette démarche nous a imposé un petit détour lorsqu'il s'agissait de comparer la fréquence absolue des deux verbes, dans la mesure où il fallait d'abord extrapoler les chiffres pour les requêtes $<$ tourner + nom $>$ sur l'ensemble du corpus. Notons que nous avons corrigé les erreurs d'étiquetage (adjectif / nom) dans notre base de données, mais les proportions entre les items initialement étiquetés 'adjectif' et 'nom' ont été respectées dans les extrapolations pour $<$ tourner + nom> (voir les notes 3 et 24). Notons encore à propos de la catégorisation que bon nombre des cooccurrents de virer et tourner ont un statut catégoriel ambigu (nom ou adjectif). Il s'ensuit qu'un certain nombre de partis pris se sont imposés. Ainsi, nous avons considéré comme des noms les N-ADJ référant à des convictions idéologiques (blairiste, gauchiste...) ou des maladies mentales (mythomane, maniaco-dépressif, etc.), du moins lorsque celles-ci se combinaient avec un sujet humain. En outre, les mots dénotant des couleurs ont toujours été considérés comme des adjectifs, y compris les noms composés (bleu clair) et les noms récemment convertis en adjectifs de couleur (framboise, crème), à l'exception des composés du type couleur $X$. Si ces conventions d'annotation peuvent paraitre arbitraires, l'essentiel est qu'elles aient été appliquées de manière cohérente aux deux verbes pour que les chiffres soient comparables.

Après nettoyage, le nombre total des attestations pertinentes des constructions à attribut du sujet se réduisait à 2791 pour virer et 894 pour tourner. De nos jours, virer attributif (2 791 attestations) est presque deux fois plus fréquent que tourner attributif (1 583 occurrences après extrapolation). En évaluant ces chiffres à la lumière de l'évolution diachronique décrite par Van Wettere et Lauwers (2017) sur la base du corpus Frantext, on constate que virer $\left({ }^{\circ} 1926\right)$, entré plus tardivement dans l'orbite attributive, y a donc supplanté tourner $\left({ }^{\circ} 1862\right)$, qui était encore plus fréquent dans la période 1926-1997 : 77,0 \% (57 attestations) vs 23,0\% (17 attestations).

Notons que, si pour la méthodologie nous avons puisé dans la boîte à outils de la Grammaire des Constructions (notamment l'analyse collostructionnelle, voir section 
3.2.1) et, de manière plus générale, de la grammaire fondée sur l'usage, nous nous abstenons ici de toute tentative de formalisation en ce sens, étant donné que l'étude de la synonymie (l'objectif principal de cette contribution) peut facilement s'en passer. Le recours à des techniques statistiques augmente le bénéfice qu'on peut tirer de l'étude de données d'observation (non élicitées, donc) en grande quantité. Elles nous permettent notamment de prendre en considération la fréquence globale des cooccurrents dans l'identification des cooccurrents qui sont vraiment attirés par le mot en question. En effet, on comprend aisément que si Paris est plus fréquemment utilisé comme sujet d'une phrase copule avec être que Saint-Pol-sur-Ternoise, cela n'a rien à voir avec une propriété spécifique du verbe être. En outre, les valeurs de signification statistique qui font office de scores d'attirance nous permettent de ne retenir que les lexèmes vraiment distinctifs dans la comparaison des deux verbes.

Afin de mieux situer les emplois attributifs, nous ferons d'abord le relevé des différentes constructions attributives (et pseudo-attributives) des deux verbes (section 2). Ensuite, nous nous focaliserons sur les emplois à attribut du sujet (au sens de 'devenir'), exemplifiés en (1) et (2). Dans un premier temps, nous chercherons à dégager des ressemblances entre les profils distributionnels des deux verbes (section 3), avant de faire apparaitre des contrastes (section 4), que nous essaierons de rattacher à leur sémantisme global. Enfin (section 5), nous montrerons que l'emploi proprement attributif des deux verbes doit être distingué d'une autre construction, identique en surface, mais dotée d'un sémantisme directionnel abstrait (exprimant une inclination ou une orientation) et de propriétés morphosyntaxiques différentes. En guise de conclusion (section 6), nous résumerons les principaux acquis de cette étude.

\section{TOURNER ET VIRER ATTRIBUTIFS : SURVOL DES CONSTRUCTIONS ET DES SENS}

Les constructions à attribut du sujet qui font l'objet du présent article ne représentent en fait qu'une partie du spectre des emplois attributifs (et pseudo-attributifs) de tourner et virer ${ }^{2}$. Pour en donner une idée, nous proposons un petit aperçu provenant de nos requêtes $\{$ virer / tourner $\}+\{\mathrm{N} / \mathrm{ADJ}\}$.

i) emplois transitifs à attribut de l'objet (AO) essentiel:

(3) si tu vires sépia ton négatif, tu ne pourras plus jamais avoir un vrai noir et blanc

(Sketch Engine)

(4) le rayon ultra-violet peut detruire l'acide amine pour tourner jaune [sic] à defraîchi les cheveux.

(Sketch Engine)

Ici virer se combine avec un COD (ton négatif) et un AO (sépia), qui s'accorde en genre et en nombre avec le COD (à chaque fois que sa forme - adjectivale - le

\footnotetext{
${ }^{2}$ Les deux verbes se combinent aussi avec le préfixe itératif : La belle tiendrait sa parole et revirerait brune par la suite (Sketch Engine). Pour retourner, v. Grevisse et Goosse (2007 : 263).
} 
permet). L'AO marque un changement d'état qui se réalise à travers le processus verbal.

ii) emplois pronominaux:

(5) pour voir si les cellules cutanées se sont tournés [sic] malignes (Sketch Engine)

Dans ces emplois pronominaux, le COD est remplacé par le pronom réflexif qui, parfois, entraîne la détransitivisation du verbe, comme dans (5), où l'alternance avec la construction (i) ( $X$ a tourné les cellules malignes) s'est perdue. Malignes s'y analyse comme un attribut du sujet.

Ces deux premières constructions (i)-(ii) sont franchement marginales dans le corpus $^{3}$.

iii) emplois à complément d'objet indirect (« pseudo-attributif»):

(6) L'entêtement vire à l'obsession chez Nicolas Sarkozy

(Sketch Engine)

La troisième construction est nettement plus fréquente, comme il ressort d'une requête (lexicale) complémentaire : les 1666 attestations de virer à et les 291 occurrences de tourner à, dans un échantillon de 15000 exemples issu d'une recherche lexicale (virer/tourner) parcouru manuellement, donnent un total extrapolé de respectivement 22150 (virer à) et 34142 attestations (tourner à) pour l'ensemble du corpus. Elle ressemble à première vue à une construction à attribut du sujet, mais, qu'on ne s'y méprenne pas, syntaxiquement parlant, il s'agit plutôt d'un complément d'objet indirect. Ainsi, la préposition est régie par le verbe (et spécifique pour celui-ci) et ne fait donc pas partie d'un constituant attributif susceptible de se retrouver encore dans d'autres contextes attributifs, contrairement à en colère, par exemple. Le régime de la préposition est toujours un $\mathrm{SN}$, jamais un adjectif ou un nom nu, ce qui fait que l'accord en genre et en nombre ne doit pas être respecté.

iv) emplois à attribut du sujet

Notre étude se focalisera sur l'emploi à attribut du sujet (ex. (1-2)), tout en sachant que la construction (iii) a joué un rôle de premier plan dans l'émergence de la construction à attribut du sujet (v. Van Wettere et Lauwers 2017), comme il ressort aussi de l'exemple (7), qui décrit un processus de changement de couleur complexe, mêlant les deux constructions.

(7) Ça vire violet, turquoise à vert magnifique!!!

(Sketch Engine)

Au sein des emplois à attribut du sujet essentiel, il faut distinguer différents emplois. En plus de leur statut comme verbes attributifs marquant un changement

\footnotetext{
${ }^{3}$ Pour virer, la construction pronominale (ii) n'est même pas attestée et la construction à AO (i) ne compte que trois attestations (recherche étiquetée $<$ virer $+\mathrm{ADJ} / \mathrm{N}>$ ). Tourner, quant à lui, est un peu plus fréquent. Il apparait 9 fois avec un attribut du COD (i) et 19 fois avec une construction pronominale suivie d'un attribut (ii), ce qui donne respectivement un total de 28 et 48 après extrapolation sur l'ensemble du corpus.
} 
d'état (ex. (1-2)), virer et tourner connaissent un emploi dans lequel ils expriment une orientation ou une inclination (soit un emploi directionnel abstrait ${ }^{4}$ ).

(8) cette année j'ai viré manga

(Sketch Engine)

'j'ai pris goût aux mangas'

(9) on tourne extrême-droite dès 30 ans

(Sketch Engine)

Il est clair que le référent du pronom sujet ne devient pas manga, mais qu'il a commencé à se tourner vers les mangas, à y prendre goût. Pour les deux verbes, la fréquence relative de cet emploi est identique, comme on le voit dans le Tableau 1.

Enfin, virer connaît encore un troisième emploi, dans lequel il exprime un état transitoire ('être provisoirement').

(10) [...] les Brissacois imposèrent leur jeu, plus individuel que collectif, pour virer en-tête à la fin du troisième $1 / 4$ temps : 63-66 et conclure le match sur le score de 71-91.

(Sketch Engine)

Ce troisième emploi - que nous n'avons pas recensé de façon systématique - ne va plus nous occuper désormais dans la mesure où il est proche d'une locution figée. Il s'accompagne en général d'indications temporelles qui explicitent l'idée qu'il ne s'agit que d'un état momentané, appréhendé à un stade précis d'une compétition sportive, ce qui veut dire que la cause n'est pas encore gagnée, comme illustré en (11).

(11) L'équipe de France a échoué dans sa quête d'une dixième Coupe Davis. Après avoir viré en tête samedi soir, Monfils et Llodra ont perdu les deux derniers simples dimanche.

(<actualites.leparisien.fr/monfils/86>; consulté le 15/05/2016)

La locution trouve son origine dans le monde de la voile :

«Virer en tête vient du langage des régates; celui qui passe une bouée du parcours avant les autres régatiers vire en tête. Les régatiers doivent contourner une bouée marquant le parcours dans le sens précisé par le règlement de la régate. Et on dit virer une bouée. »

$<$ http://correcteurs.blog.lemonde.fr/2014/03/22/le-telesiege-enseignement> consulté le 15/06/2015

Elle a ensuite commencé à être employée métaphoriquement avant de s'étendre à d'autres expressions proches comme les numéraux ordinaux et l'adjectif invaincu ${ }^{5}$.

(12) L'équipe 2 masculine vire première à l'issue des matches allers

<www.club.fft.fr/...d/.../infotennisdecembre2013.pdf> consulté le 15/06/2015

(13) Et voilà comme le club espagnol vire invaincu, au terme de ces matches aller.

(Sketch Engine)

Notons que l'attribut s'accorde avec le sujet et qu'il ne peut pas être omis sans changer le sens du verbe, qui tend à abandonner sa valeur dynamique : on glisse de 'passer' à 'être'. Nous avons ici affaire à un cas de figure analogue à finir

\footnotetext{
${ }^{4}$ Le profil distributionnel de ces emplois directionnels abstraits sera discuté à la section 5 , tout comme leurs propriétés syntaxiques et sémantiques.

${ }^{5}$ Sur cette expression se greffent également des attributs accessoires : La 19eme journée de Ligue 1 a vu le PSG virer invaincu en tête <www.footafrica365.fr/.../article_743106_africainsde-france-11-Le-tablea...> consulté le 21/12/2016.
} 


\begin{tabular}{lccl}
\hline \hline & changement d'état 'devenir' & inclination / orientation & Total \\
\hline Tourner & $94,5 \%(845)$ & $5,5 \%(49)$ & $100 \%(894)$ \\
Virer & $94,5 \%(2637)$ & $5,5 \%(154)$ & $100 \%(2791)$ \\
\hline \hline
\end{tabular}

Tableau 1: Changement d'état vs inclination / orientation

premier (passer premier, faire deuxième, etc.; v. Lauwers et Tobback 2010). En effet, la charge prédicative se déplace vers l'attribut. On assiste donc à un processus de réanalyse sémantique au bout duquel l'attribut accessoire (en tête/premier/...), référant à l'état dans lequel le référent du sujet se trouve pendant l'action, devient un attribut essentiel. Désémantisation du verbe et présence obligatoire (angl. obligatorification) de l'attribut vont de pair ici.

\section{TOURNER / VIRER + ATTRIBUT DU SUJET : CONVERGENCES}

Il convient tout d'abord d'examiner ce que les deux verbes ont en commun lorsqu'ils se combinent avec un attribut du sujet. Dans ce qui suit, nous établirons dès lors le profil sémantique global des deux verbes sur la base de leur profil distributionnel (section 3.2.). Pour établir le profil distributionnel, nous nous servirons de l'analyse collexémique simple développée par Stefanowitsch et Gries (2003) et Gries et Stefanowitsch (2004), dont nous rappellerons d'abord les principes (section 3.1).

\subsection{Analyse collexémique simple : méthodologie}

La méthode collostructionnelle, comme toute application dans le domaine de la sémantique distributionnelle ${ }^{6}$, est basée sur l'adage " You shall know a word by the company it keeps » (Firth 1957 : 11). En effet, les contextes dans lesquels un mot est employé nous fournissent des indices précieux pour capter certaines distinctions sémantiques parfois subtiles au sein d'un ensemble de mots sémantiquement apparentés, qui ne sont pas accessibles / objectivables si l'on se fonde uniquement sur ses intuitions sémantiques. Si la comparaison de cooccurrents et la prise en compte de leur fréquence brute (raw frequencies) constituent déjà des stratégies très utiles, celles-ci gagnent encore en puissance si l'on parvient à objectiver statistiquement les préférences distributionnelles par le biais d'une analyse de probabilités. Parmi les nombreuses méthodes possibles, nous avons choisi la méthode collostructionnelle développée par Stefanowitsch et Gries (2003). Conçue dans l'esprit de la

\footnotetext{
${ }^{6}$ Nous utiliserons donc la fréquence / la distribution comme une approximation de la façon dont les locuteurs conceptualisent le sens (à compléter avec d'autres facteurs tels que la saillance). L'enjeu de l'article n'étant pas de nature méthodologique, nous nous limiterons ici à signaler que des corrélations encourageantes ont été observées entre la distribution (et la fréquence) et les intuitions des locuteurs (telles que celles-ci sont mesurables à travers des expériences comportementales). Voir, entre autres, Heylen et al. (2015) et Perek (2016).
} 
grammaire des constructions, elle offre un modèle d'interprétation qui se prête directement à l'analyse d'outils grammaticaux tels que les verbes attributifs. Concrètement, elle prend en considération la notion de construction, et notamment le degré d'association entre une position (un slot) dans une construction donnée et les items lexicaux occupant cette position, les collexèmes. De ce fait, elle s'avère plus précise et plus fiable que la plupart des analyses collocationnelles plus traditionnelles, qui sont basées en général sur l'extraction automatique de listes de lexèmes cooccurrents dans une fenêtre de $n$ mots (Stefanowitsch et Gries 2003 : 210), une approche communément appelée « bag of words model ». En outre, l'assise statistique (à savoir le Fisher Exact Test) sur laquelle repose cette méthode permet l'application à des ensembles de données de taille relativement modeste. Elle a cependant comme désavantage - à côté des désavantages classiques de toute approche distributionnelle du sens (comme la négligence de la polysémie) ${ }^{7}$ - d'être plus consommatrice en temps (tri manuel des données), désavantage qui est en partie compensé par l'existence de scripts sous $\mathrm{R}^{8}$ (Gries 2014), qui permettent d'accélérer l'exécution des calculs statistiques (pour un bilan récent, voir Stefanowitsch 2013).

Cette méthode, qui connait un certain nombre de variantes et d'extensions - dont l'analyse collexémique distinctive (v. section 4.1) -, permet donc de mesurer le degré d'association entre une construction (ou plusieurs constructions) et les lexèmes qui $\mathrm{y}$ entrent $(=$ collexèmes $)$. Le principe est très simple : un lexème $\mathrm{X}$ apparait-il plus fréquemment dans une position d'une construction $\mathrm{C}$ (en l'occurrence virer ou tourner + attribut) que ce que prédit le hasard? Pour savoir ce que prédit le hasard, il faut prendre en considération les fréquences attendues/théoriques telles que les fréquences globales dans l'ensemble du corpus permettent de les établir. Autrement dit, pour chaque cooccurrent (collexème) il faut établir un tableau croisé ou de contingence, ce qui, pour un corpus d'un million de mots, donne la situation dans le Tableau 2.

Dans la méthode collostructionnelle, le degré d'association (association strength) est directement déduit de la valeur $p$ du Fisher Exact Test. Si la probabilité que la distribution observée est due au hasard est inférieure à 0.05 ( $p<$ $0.05)$, on est en droit de conclure que l'association est significative. Autrement dit, la valeur $p$ est utilisée par Gries et Stefanowitsch (2003) comme une mesure du degré d'association.

Une fois les valeurs $p$ obtenues, il suffit de les classer dans l'ordre croissant / décroissant. Pour faciliter l'interprétation des résultats, on utilise en général une transposition logarithmique des valeurs $p$ du Fisher Exact Test (-log10). Ainsi, un haut degré d'association correspond désormais à une valeur élevée (plutôt qu'à une valeur $p$ très basse).

Comme l'analyse collexémique simple se fonde sur la fréquence globale des lexèmes en position d'attribut du sujet dans le corpus Sketch Engine, nous avons dû exclure de l'analyse collostructionnelle les items polylexicaux (noms composés,

\footnotetext{
${ }^{7}$ Ce problème peut cependant être résolu si l'on passe du niveau du mot (type) au niveau de l'occurrence (token). Voir Heylen et al. (2015).

${ }^{8}<$ http://www.linguistics.ucsb.edu/faculty/stgries/>, consulté le 15/06/2015. Voir Gries (2014).
} 


\begin{tabular}{lcc}
\hline \hline & dans construction C & dans d'autres constructions (-C) \\
\hline fq lexème X & 100 & $5000^{\mathrm{a}}$ \\
fq autres lexèmes (-X) & 500 & $994400^{\mathrm{b}}$ \\
\hline \hline
\end{tabular}

${ }^{\mathrm{a}}$ fréquence de $\mathrm{X}$ ailleurs; ${ }^{\mathrm{b}} n$ total des occurrences du corpus - la somme des 3 autres cellules du Table

Tableau 2: Table de contingence (analyse collexémique 'standard')

prénom + nom, etc.) qui n'étaient pas reconnus en tant que tels par le logiciel d'interrogation. Il s'agit de 185 occurrences pour virer et 24 pour tourner. Vu qu'il s'agit pour la plupart d'hapax, leur impact pour le profil sémantique de la construction attributive est négligeable.

Notons que nous avons d'emblée procédé à une analyse unitaire, ne distinguant pas entre noms et adjectifs, la distinction étant d'ailleurs assez délicate, voire non pertinente, pour bon nombre d'items ambivalents (p. ex. communiste). La distinction n'est d'ailleurs pas déterminante, car on obtient des résultats sensiblement analogues si l'on effectue les analyses séparément.

\subsection{Résultats}

Pour faire ressortir les similarités entre les profils sémantiques des deux verbes établis à partir de leurs collexèmes les plus significatifs, nous allons regrouper ceux-ci en classes sémantiques (section 3.2.2). Mais avant cela, il convient de présenter les collexèmes mêmes - donc les types -, ainsi que leur fréquence d'apparition (token frequency), ce qui nous amènera aussi à conclure à une certaine tendance au figement (section 3.2.1).

\subsubsection{Types (= collexèmes) partagés et figement}

À regarder le nombre de lexèmes (= les types) cooccurrents partagés par les deux verbes, on constate qu'à peine $13 \%(12,96 \%)$ des collexèmes (hors 'inclination') sont partagés par les deux verbes (134 sur 1034 ). Ce chiffre est cependant doublement trompeur. Il est tout d'abord biaisé par la différence entre le nombre d'occurrences vues. Si nous avions pu analyser autant d'occurrences de tourner que de virer, nous aurions constaté que bon nombre des hapax de virer apparaissent aussi avec tourner, ce qui aurait augmenté le taux de recouvrement. On peut neutraliser ce biais en faisant abstraction des hapax : dans ce cas, 134 des 307 non-hapax sont partagés, soit 43,6\%. En plus, ce chiffre de $13 \%$ cache le fait que les types partagés sont très fréquents. En effet, le recouvrement en termes d'occurrences (tokens) des types partagés se monte jusqu'à 61,99 \% (2 159 / 3 483).

Que le taux de recouvrement des deux verbes soit plus élevé ressort aussi de l'analyse du top 100 des collexèmes les plus attirés (cf. l'analyse collexémique simple, section 3.2.2) : 25 des 90 items (100 - la catégorie VII) les plus attirés sont partagés par les deux verbes. Cela signifie que les locuteurs entendent souvent les mêmes items associés aux mêmes verbes, étant donné que la fréquence 
des collexèmes les plus attirés est en général plus élevée (Zeschel 2012), d'où l'impression d'une synonymie quasi parfaite dans le chef des locuteurs'.

Le fait que les types les plus attirés (partagés ou non) soient en général aussi assez fréquents nous amène à un autre constat : les deux verbes font preuve d'une certaine tendance à la constitution de collocations, voire même au figement. Ainsi, le cooccurrent le plus important est le même pour les deux verbes : virer fou (504 attestations sur 2 791) et tourner fou (167 sur 894), avec des extensions vers des adjectifs sémantiquement proches comme dingue/dingo/dinguo et maboul. Tout indique que nous avons ici affaire à des extensions analogiques dans le sens de Bybee et Eddington (2006) : « novel instances of verb + adjective sequences are based on analogies to previous experience ». Ces extensions nous semblent d'autant plus probables qu'elles se rapportent à un cooccurrent prototypique. En outre, dans le cas de virer, fou entre dans une sorte de formule impersonnelle passe-partout signifiant 'il ne faut pas exagérer, il faut raison garder'.

(14) Et pour tous ces craintifs de punaises de lit : oui, il y a un problème d'insectes. Mais faut pas virer fou. Ce n'est pas en mettant le pied sur l'île de Manhattan que vous allez être dévorés vivants

(Sketch Engine)

Cette expression fournit la plupart des 132 exemples sans sujet grammatical. Du côté de tourner fou, on note la présence de l'expression quasi adverbiale \{la vis/la machine/la roueletc. $\}$ tourne $\{$ fou/folle $\}$ 'dans le vide'. Celle-ci n'accepte pas la commutation par d'autres verbes copules et le verbe y est encore moins désémantisé (la vis tourne effectivement). Seul l'accord montre que le tour tend à être réanalysé comme locution attributive.

De même, vinaigre et chèvre - et aussi bourrique pour tourner - constituent des expressions figées (ou ne permettant que très peu de variation : (en) bourrique).

(15) un niaf qui s'marre la tronche à me faire virer chèvre et tourner bourrique

(Sketch Engine)

Ces noms n'ont pas pour autant cessé de fonctionner comme des attributs du sujet, car l'accord est possible, ce qui est confirmé par les estimations fournies par Google (10-06-2016) pour les sujets pluriels (voir le Tableau 3).

Enfin, d'autres groupes de quasi-synonymes associés conventionnellement à l'un des deux verbes s'observent (tourner vilain, virer pivoine); ils seront discutés dans le cadre de l'analyse sémantique (section 3.2.2).

L'impression du recouvrement considérable des cooccurrents attributifs des deux verbes est confirmée si l'on regroupe les types en (six) classes sémantiques. C'est ce que nous allons montrer maintenant.

\footnotetext{
${ }^{9}$ Le taux de recouvrement lexical est cependant inférieur à celui observé pour un autre couple de verbes attributifs s'avérer / se révéler (Tobback et Lauwers 2012) qui partagent 120 de leurs 478 collexèmes, soit $25,1 \%$ des types (corpus journalistique), correspondant à $66,0 \%$ des occurrences. Si l'on fait abstraction des hapax, le taux de recouvrement atteint $69,3 \%$ des types (120 des 173 types non hapax).
} 


\begin{tabular}{lrrr}
\hline \hline support pluriel & attribut singulier & attribut pluriel & \multicolumn{1}{c}{ TOTAL } \\
\hline «virent chèvre(s)» & Sketch Engine $: 0$ & Sketch Engine $: 1$ & Sketch Engine $: 1$ \\
& Google $: 1$ & Google $: 3$ & Google $: 4$ \\
«tournent chèvre(s)» & Sketch Engine $: 3$ & Sketch Engine $: 1$ & Sketch Engine $: 3$ \\
& Google $: 8$ & Google $: 19$ & Google $: 27$ \\
«tournent bourrique(s)» & Sketch Engine $: 4$ & Sketch Engine : 0 & Sketch Engine $: 4$ \\
& Google $: 12$ & Google $: 6$ & Google $: 18$ \\
\hline \hline
\end{tabular}

Tableau 3: Accord verbe/sujet Sketch Engine et Google

\subsubsection{Classes sémantiques partagées : le caractère défavorable du changement d'état}

Pour chaque verbe, la sortie du script se présente comme suit. Notons que nous nous limitons ici au top dix des collexèmes les plus attirés (Tableaux 4 et 5).

Ces tableaux doivent être lus de la manière suivante : fréquence totale du mot dans le corpus; fréquence observée; fréquence attendue (théorique); \% des occurrences du mot qui apparaissent avec le verbe en question (« faith »); l'attraction unidirectionnelle : dans quelle mesure le verbe permet-il de deviner le mot (« delta.p. constr.to.word $»)^{10}$ et dans quelle mesure peut-on prédire l'apparition du verbe à partir du mot (« delta.p.word to construction »)?; degré d'association. Seule la dernière colonne est pertinente pour ce qui suit. Les items les plus attirés se trouvent dès lors au sommet du tableau et certains sont à ce point attirés par le verbe en question que la chance de se tromper est infiniment petite ( $«$ Inf »).

Que peut-on conclure de ces résultats? Dans l'analyse collostructionnelle simple, le degré d'association et donc le rang occupé par l'item en question est plus important que le seuil même de $1.30103(\sim p<0.05)$, car peu d'items ne sont pas attirés de manière significative par les verbes (respectivement 115 items pour virer et 12 pour tourner). C'est pourquoi les analyses se concentrent toujours sur les collexèmes les plus fortement attirés. Dans (16)-(23), nous nous limiterons dès lors au top 100 des collexèmes, que nous avons ensuite regroupés en catégories sémantiques (qui ont une identité suffisamment nette pour qu'elles soient accessibles à l'introspection); leur rang a été indiqué entre parenthèses.

\footnotetext{
${ }^{10}$ Plus précisément, « $\Delta \mathrm{P}$ est la probabilité d'obtenir le résultat sachant que l'indice est présent $\mathrm{P}(\mathrm{O} \mid \mathrm{C})$ moins la probabilité du résultat en l'absence de l'indice $\mathrm{P}(\mathrm{O} \mid \neg \mathrm{C})$. Lorsque ces probabilités sont identiques, il n'y a pas de covariation entre les deux événements. On considère que plus $\Delta \mathrm{P}$ est proche de 1.0 , plus la présence de l'indice augmente la probabilité du résultat, alors que plus $\Delta \mathrm{P}$ est proche de -1.0 , plus la présence de l'indice diminue la probabilité du résultat » (Ellis 2006 : 11, cité et traduit dans Desagulier 2015 : 116).
} 


\begin{tabular}{|c|c|c|c|c|c|c|c|}
\hline \multicolumn{8}{|c|}{ Virer } \\
\hline words & $\begin{array}{l}\text { word. } \\
\text { freq }\end{array}$ & $\begin{array}{l}\text { obs. } \\
\text { freq }\end{array}$ & $\begin{array}{l}\text { exp. } \\
\text { freq }\end{array}$ & faith & $\begin{array}{l}\text { delta.p. } \\
\text { constr.to. } \\
\text { word }\end{array}$ & $\begin{array}{l}\text { delta.p. } \\
\text { word.to. } \\
\text { constr }\end{array}$ & $\begin{array}{l}\text { coll. } \\
\text { strength }\end{array}$ \\
\hline fou & 1568060 & 500 & 0.4134 & $3,00 \mathrm{e}-04$ & 0.1916 & $3,00 \mathrm{e}-04$ & Inf \\
\hline parano(ïaque) & 40204 & 104 & 0.0106 & 0.0026 & 0.0399 & 0.0026 & Inf \\
\hline rouge & 1441704 & 124 & 0.38 & $1,00 \mathrm{e}-04$ & 0.0474 & $1,00 \mathrm{e}-04$ & 2.607.276 \\
\hline dingo/gue & 100394 & 54 & 0.0265 & $5,00 \mathrm{e}-04$ & 0.0207 & $5,00 \mathrm{e}-04$ & 1.567 .966 \\
\hline gay & 29142 & 39 & 0.0077 & 0.0013 & 0.015 & 0.0013 & 1.289.143 \\
\hline pivoine & 7910 & 24 & 0.0021 & 0.003 & 0.0092 & 0.003 & 881.956 \\
\hline lesbienne & 20896 & 24 & 0.0055 & 0.0011 & 0.0092 & 0.0011 & 780.623 \\
\hline écarlate & 23056 & 24 & 0.0061 & 0.001 & 0.0092 & 0.001 & 770.367 \\
\hline facho/fasciste & 61754 & 26 & 0.0163 & $4,00 \mathrm{e}-04$ & 0.01 & $4,00 \mathrm{e}-04$ & 731.668 \\
\hline hystéro/rique & 3186 & 17 & $8,00 \mathrm{e}-04$ & 0.0053 & 0.0065 & 0.0053 & 668.812 \\
\hline
\end{tabular}

Tableau 4: Virer (analyse collexémique standard) 


\begin{tabular}{|c|c|c|c|c|c|c|c|}
\hline \multicolumn{8}{|c|}{ Tourner } \\
\hline words & $\begin{array}{l}\text { word. } \\
\text { freq }\end{array}$ & $\begin{array}{l}\text { obs. } \\
\text { freq }\end{array}$ & $\begin{array}{l}\text { exp. } \\
\text { freq }\end{array}$ & faith & $\begin{array}{l}\text { delta.p. } \\
\text { constr. } \\
\text { to. } \\
\text { word }\end{array}$ & $\begin{array}{l}\text { delta.p. } \\
\text { word.to. } \\
\text { constr }\end{array}$ & $\begin{array}{l}\text { coll. } \\
\text { strength }\end{array}$ \\
\hline fou & 623345 & 168 & 0.0548 & $3,00 \mathrm{e}-04$ & 0.193 & $3,00 \mathrm{e}-04$ & Inf \\
\hline vinaigre & 65237 & 41 & 0.0057 & $6.00 \mathrm{e}-04$ & 0.0471 & $6.00 \mathrm{e}-04$ & 141.836 \\
\hline bourrique & 7506 & 31 & $7.00 \mathrm{e}-04$ & 0.0041 & 0.0356 & 0.0041 & 132.765 \\
\hline aigre & 8657 & 24 & $8.00 \mathrm{e}-04$ & 0.0028 & 0.0276 & 0.0028 & 98.785 \\
\hline chèvre & 83858 & 28 & 0.0074 & $3.00 \mathrm{e}-04$ & 0.0322 & $3.00 \mathrm{e}-04$ & 893.791 \\
\hline maboul & 2239 & 16 & $2.00 \mathrm{e}-04$ & 0.0071 & 0.0184 & 0.0071 & 72.694 \\
\hline rouge & 1441704 & 34 & 0.1268 & 0 & 0.0389 & 0 & 692.962 \\
\hline $\begin{array}{l}\text { parano } \\
\text { (iaque) }\end{array}$ & 40204 & 17 & 0.0035 & $4.00 \mathrm{e}-04$ & 0.0195 & $4.00 \mathrm{e}-04$ & 56.296 \\
\hline dingo/gue & 100394 & 14 & 0.0088 & $1.00 \mathrm{e}-04$ & 0.0161 & $1.00 \mathrm{e}-04$ & 397.454 \\
\hline bleu & 958769 & 17 & 0.0843 & 0 & 0.0194 & 0 & 329.105 \\
\hline
\end{tabular}

Tableau 5: Tourner (analyse collexémique standard) 
(16) Etat de faiblesse mentale

a. Folie (et stupidité) :

Virer

fou (1), dingo/gue (4), chèvre (26), maboul (28), barge (29), mémè(re) (47), barjo(t) (51), niais (64), mongol (82)

+ bizarre (90)

b. État de maladie mentale:

Virer

parano(iaque) (2), hystéro/rique (10), schizo(phrène) (11), psychopathe (21), dépressif (32), maniaque (41), geek (44), misanthrope (46), sado-maso (50), gaga (61)

c. État maladif de dépendance :

Virer alcoolo/lique(13), anorexique (43), boulimique (59), couchomaniaque (67), claustro (68), maso(chiste) (92), mytho(mane) (96), addict (98)

+ clodo (84)

+ émo(tionnel) (48), bisounours (99), pot-de-colle (100)

\section{(17) Convictions idéologiques}

Virer facho/fasciste (9), gauchiste (14), écolo (15), libéral (17), réac(tionnaire) (30), féministe (35), végétarien (36), sarkozyste (37), nationaliste (38), rebelle (54), fascistoïde (76), négrophobe (86), anti-sémite (55), poujadiste (60), démocrate (70), bouddhiste (71), néocon(servateur) (73), anar(chiste) (74), extrême-droite (80), socialo/liste(81), collabo(rateur) (85), ultralibéral (88), catho(lique) (97)

+ liées à un style de vie: mystique (18), goth(ique) (27), hippie (56), punk (76), bobo (95), moine (93)

(18) Orientations sexuelles

Virer

gay (5), lesbienne (7), pédé (19), hétéro (22), homo(sexuel) (39), herma(phrodite) 'plantes' (53), travelo (77)

\section{Tourner}

fou (1), bourrique (3), chèvre (5), dingo/gue (9), fada (12), sot (14), barge (29), délirant (73), follasse (78), frapadingue (81),

+ bizarre (16), weird (92)

\section{Tourner}

parano(ïaque) (8), alter-trisomique (37), sénile (47), gaga (52), psycho (56), schizo(phrène) (58), hystéro/rique (71)

\section{Tourner}

alcoolo/lique (77)

\section{Tourner}

bolchévique (23), végétarien (31), facho/fasciste (55), social-capitaliste (62), Sarko-compatible (72), réac(tionnaire) (74), raciste (89), néocolonialiste (91), péripatéticien (95), communiste (97)

+ liées à un style de vie: mystique (46), goth(ique) (82)

\section{Tourner}

gay (13), lesbienne (19), homo(sexuel) (24), pédé (27), gaillouffe (38), herma(phrodite) 'plantes' (42), hétéro (67), goudou (85), zoophile (94) 
(19) Comportements humains détestables

Virer

Tourner

méchant (31), asocial (94), malhonnête (65) délinquant (30), pervers (49), con (70)

agressif (78), terroriste (79),

bling bling (89), voyou (91)

pute (83)

(20) Couleurs

Virer

rouge (3), pivoine (6), écarlate (8),

jaune (12), marron (20), bleu (23),

orange (24), écrevisse (33), violet (34),

\section{Tourner}

rouge (7), bleu (10), vert (11), jaune (15), noir (21), brun (22), blanc (34), gris (35), marron (39), violet (44), rose (45),

gris (40), vert (42), châtain (45), rose (57), sombre (40), semi-bleu (48), grisâtre (53), blanc (58), blême (69), brun (75), roux (83), cramoisi (86) jaunâtre (54), orange (61), écarlate (63), pourpre (69), pâle (88), cramoisi (100) + fadasse (32)

(21) Putréfaction / acidification / dégénération d'une situation Virer vinaigre (16), glauque (72)

\section{Tourner}

vinaigre (2), aigre (4), glauque (17), vilain (18), méchant (20), dangereux (25), laid (26), mauvais (33), vicieux (36), négatif (41), rance (43), mortel (59), cancéreux (66), chaud (68), violent (75), malsain (80), amer (87), horripilant (96), pestilentiel (98), chocolat $(99$, situation qui tourne chocolat) + nuageux (60)

(22) Domaines de préférence / centres d'intérêt [inclination]

Virer

hardcore (52), pop (62), électro (63), techno (87)

\section{(23) Autres}

Virer

positif (49)

\section{Tourner}

anti-menace (50), outre-Atlantique (51), multijoueur (64), électro (79), black-metal (84)

\section{Tourner} positif (28), haussier (57), saprophyte (90, péj.), français (65), cryptique (93)

Le constat majeur qui en émerge, c'est que les deux verbes sont caractérisés par une valeur négative. Ils expriment un changement d'état défavorable, qui affecte de manière négative - selon le locuteur/scripteur - le support de la prédication (le plus souvent le sujet). Il s'ensuit que les mots du contexte (notamment les cooccurrents à droite) sont souvent marqués par une «prosodie sémantique négative » (Sinclair 1987), c'est-à-dire qu'ils évoquent des associations négatives, à l'instar de à cause de, exemple désormais classique. Cela ressort clairement des cooccurrents les plus attirés par les verbes et des classes sémantiques auxquelles ils appartiennent. Les items se laissent facilement regrouper, notamment dans le cas de virer, où seul positif - item curieux à plus d'un titre (voir ci-dessous) - n'a pas pu être classé. Il en ressort 6 catégories sémantiques. Tout d'abord, les deux verbes se combinent 
souvent avec des noms/adjectifs qui dénotent un état de faiblesse mentale (16) (respectivement 28 et 25 types pour les deux verbes), que ce soit la folie pure et simple (fou, dingue) ou une maladie mentale plus spécifique (parfois banalisée comme paranoïaque, hystérique), y compris toutes sortes d'addictions (alcoolique, par exemple). Les convictions idéologiques (17) sont également très bien représentées (notamment dans le cas de virer; 27 types) avec, dans leur sillage, le style de vie qu'elles impliquent (bobo, goth(ique)). Les orientations sexuelles (18) - ce qui peut surprendre, car le nombre d'options (et donc de noms disponibles) est, référentiellement parlant, restreint - constituent également une classe importante. La quatrième catégorie, moins fournie que les trois autres (11 types au total pour les deux verbes), regroupe des comportements humains criminels détestables ou en tout cas répréhensibles (19). Plus importante que cette dernière, la classe des couleurs (20) fournit un total de 26 types pour les deux verbes. Cette classe se distingue clairement des classes précédentes par la nature purement physique de leur dénotation, tout comme la dernière catégorie, plus difficile à circonscrire et plus ou moins exclusivement liée à tourner (voir ci-dessous), à savoir les prédicats qui réfèrent à un état de putréfaction ou d'acidification (vinaigre, aigre, rance, putride...) ou, de manière plus générale ou métaphorique, à un état de dégénérescence ou, en tout cas, à une situation qui dégénère (la situation tourne \{vilain/laid/vinaigre\}) (21). Enfin, dans le top 100 nous avons également pu identifier une dizaine de types qui renvoient à des domaines/genres de préférence ou des centres d'intérêt (22) liés à l'emploi 'inclination'/'orientation' (section 5).

Si l'on fait pour l'instant abstraction des différences entre tourner et virer, qui seront objectivées et approfondies au moyen d'une analyse collexémique distinctive, force est de constater que les deux verbes s'associent à des changements d'état aboutissant à un état ou à une entité perçus comme étant néfastes : un état de faiblesse mentale (folie, maladies mentales, penchants maladifs), des comportements humains criminels/détestables et la putréfaction/dégénération. Le sémantisme même des cooccurrents en fait foi. Or, en plus de cela, les types représentés parmi les convictions politiques et les orientations sexuelles sont le plus souvent connotés négativement, dans la mesure où elles sont considérées comme déviantes par rapport à la norme (et le choix de variantes terminologiques vulgaires 'en rajoute' encore) :

- gay, homo(sexuel), lesbienne, pédé, travelo, gaillouffe, goudou, tantouze, zoophile, etc.

- facho/fasciste, réac(tionnaire), anti-sémite, raciste, poujadiste, anar(chiste), extrême-droite, collabo(rateur), ultralibéral, goth(ique), Sarko-compatible, fascistoïde, négrophobe, néocolonialiste, bobo, hippie, etc.

Certes, on ne peut jamais exclure que, sous la plume de certains scripteurs, ces mots prennent une connotation positive, ou que des mots comme gauchiste et catho(lique) soient associés à une connotation négative, mais la tendance est nette. Prenons-en pour preuve le sort de hétéro. En soi, hétéro couvre à lui seul tout le spectre couvert par [-gay] et [-lesbienne]. Sa fréquence dans l'ensemble du corpus Sketch Engine (53 873) dépasse dès lors de loin la fréquence de gay (29 142), lesbienne (20 896) et pédé (9 940), mais il n'empêche qu'il ne se combine que 12 fois avec 
virer, contre 39 fois pour gay, 24 pour lesbienne et 10 pour pédé. En outre, pour virer hétéro, le contexte de 7 des 12 attestations montre que le changement est vu comme négatif. Il suffit de penser à certains discours homophobes qui mettent l'homosexualité en rapport avec la maladie (psychique), ou encore, à l'idée que certaines convictions sont le fait d'esprits malades, pour faire apparaitre les liens entre les trois premières catégories sémantiques.

Cette prosodie sémantique négative serait-elle pour autant absolue? Comme il est impossible de juger de l'état d'âme du scripteur à partir du seul contexte linguistique, et cela pour tous les exemples, il est plus utile de se tourner vers les termes incontestablement associés à une connotation positive ou neutre figurant dans le top 100 des collexèmes (laissant pour l'instant de côté les couleurs, dont certaines sont tout sauf neutres par rapport à l'axe bénéfique/défavorable).

La présence du lemme positif aux rangs 49 (virer) et 28 (tourner) a de quoi interpeller, d'autant plus qu'il s'agit d'un mot très fréquent (respectivement 13 et 6 attestations), qui s'avère même plus fréquent que son antonyme négatif, davantage attendu ici (virer : rang 822, 1 attestation; tourner : rang 41, 4 attestations). Les contextes d'emplois font toujours apparaitre des tests et, par extension métonymique, des personnes effectuant les tests (la femme avait viré positif pour le CMV) ou les résultats d'un tel test (résultats négatifs depuis autant d'années peuvent virer positif), qui affichent un résultat positif, souvent lié à un changement de couleur du dispositif test. Le paradoxe s'explique de deux manières. Tout d'abord, 'positif' signifie ici souvent (mais pas toujours) 'fâcheux' pour celui qui subit le test, ce qui confirme l'orientation péjorative des verbes. En outre, on pourrait y voir la survie du contexte dans lequel virer attributif a émergé (v. Van Wettere et Lauwers 2017) à savoir un contexte chimique dans lequel une solution opère un changement de couleur sur le dispositif test (un papier tournesol qui vire) ${ }^{11}$. En revanche, tourner haussier, expression relevant du jargon boursier (tourner : rang 57), constitue un contre-exemple moins équivoque.

Enfin, notons encore la présence d'une série de termes a priori neutres, qui sont cependant utilisés dans un contexte qui montre clairement que le scripteur regrette le changement d'état en question ([plante] hermaphrodite, mystique, saprophytes, moine, bisounours, catho(lique) $)^{12}$. Pour trouver des termes neutres, il faut descendre bien au-delà du rang 100 : avocat (tourner, rang 167), géographe (économique et politique) (tourner, rang 267). Il n'en reste pas moins que souvent le contexte témoigne d'une certaine ironie dans l'expression, le statut résultant étant un peu absurde ou, en tout cas, inattendu pour le scripteur.

(24) En tant qu'ancien directeur financier puis DG d'une multinationale conquérant tout [sic] les continents (j'exagère un peu) et qui a tourné géographe (économique et politique), j'ai attaqué avec appétit l'article de Laurent Carroué

(Sketch Engine)

${ }^{11}$ L'association avec l'emploi intransitif sans attribut (virer 'changer de couleur') pourrait renforcer la thèse d'une filiation diachronique locale et indépendante de ce secteur particulier du spectre attributif de virer (et de son pendant indirect : virer à + couleur).

${ }^{12}$ Le fait que Wilmet (2007 : 517) signale que virer malhonnête signifie 'donner dans la délinquance' suggère que le verbe en lui-même renforce l'orientation négative de l'adjectif. 
(25) le cœur du Colonel Sarah McKenzie appartenait à jamais à cet aviateur tourné avocat. Et en retour, le cœur de l'avocat était totalement dédié à l'intrépide Marine

(Sketch Engine)

En définitive, il n'est pas impossible de trouver des contextes neutres, voire positifs, mais ceux-ci restent très marqués et se prêtent souvent à des exploitations ironiques.

L'orientation défavorable du changement d'état marqué par les deux verbes n'apparait pas uniquement dans le choix des noms et des adjectifs constituant la tête du syntagme attributif. On note aussi la présence d'adjectifs subjectifs antéposés péjoratifs comme gros (virer \{gros bill, gros rock, gros melon, gros son sans intérêt, grosse boite impersonnelle, grosse daube\}; tourner $\{$ grosse pute du politic-bizness $\}$, etc.), vieux (virer $\{$ vieux con, vieille conne, vieille croulante, vieille fille, vieux croûton, vieux garçon\}; tourner $\{$ vieux con\}), mauvais (virer/ tourner mauvais garçon; virer bad boy; virer mauvaise langue; virer mauvais goût) ou des préfixes comme ultra (virer \{ultralibéral, ultra-gauchiste, ultra bayrouiste, ultra catho, ultra religion, ultra-jaloux\}) qui indiquent la transgression d'une norme. Enfin, notons encore la présence d'autres éléments contextuels qui accompagnent les deux verbes indiquant que l'état résultant du changement est à éviter (risquer de, pour ne pas, etc.).

Notons, pour finir, qu'en plus d'un sémantisme péjoratif, les cooccurrents témoignent du registre familier ${ }^{13}$, voire populaire, auquel appartiennent ces deux verbes. Pensons aux désignations de la folie (maboul, dingue, fada...) et les multiples synonymes vulgaires pour homosexuel et lesbienne, par exemple. Le relevé des cooccurrents reflète plusieurs procédés de la langue familière / populaire tels que la troncation (intello, facho/gaucho, psycho, émo, goth(ique), macho, anar, réac, etc.), combinée parfois à l'affixation en $-o(s)$ (terros 'terroristes', dinguo, etc.), le verlan (barjo, ripoux, etc.), les emprunts à l'anglais, voire des mots anglais (gay-friendly, geek, weird, no-life addict) et toutes sortes de formations créatives (barbu-macho, couchomaniaque 'maniaque de langes', pipeule-nichons, etc.). On ne saurait cependant croire que ces deux verbes restent cantonnés au registre familier, car bon nombre des cooccurrents proviennent de la presse écrite (p. ex. sportive) et peuvent passer pour des termes neutres (voire plutôt formels dans certains cas : astigmate, centriste).

\section{TOURNER VERSUS VIRER : VARIATIONS SUR LE THÈME DU CHANGEMENT D'ÉTAT}

Jusqu'ici nous nous sommes focalisés sur ce que ces deux verbes ont en commun. Toutefois, certaines différences se sont déjà manifestées en filigrane, comme par exemple la prédilection de virer pour les sujets humains, là où tourner se combine aussi bien avec des sujets humains que des sujets inanimés (chi carré 346.19, $d f=$ $1, p=2.2 \mathrm{e}-16$; après exclusion des cas indécis, où le contexte ne permet pas de

\footnotetext{
${ }^{13} \mathrm{Il}$ n'est pas à exclure que certaines variantes régionales du français (notamment le français québécois) soient mieux représentées ici que d'autres. Notre corpus web ne permet pas d'en juger.
} 


\begin{tabular}{|c|c|c|c|c|c|c|c|c|c|c|}
\hline & \multicolumn{2}{|c|}{ animal } & \multicolumn{2}{|c|}{ humain } & \multicolumn{2}{|c|}{ inanimé } & \multicolumn{2}{|c|}{ indécis } & \multicolumn{2}{|c|}{ TOTAL } \\
\hline & $\mathrm{n}$ & $\%$ & $\mathrm{n}$ & $\%$ & $\mathrm{n}$ & $\%$ & $\mathrm{n}$ & $\%$ & $\mathrm{n}$ & $\%$ \\
\hline Tourner & 14 & 1,57 & $483^{\mathrm{a}}$ & 54,03 & 396 & 44,3 & 1 & 0,11 & 894 & 100 \\
\hline Virer & 29 & 1,04 & $2346^{\mathrm{b}}$ & 84,06 & 414 & 14,83 & 2 & 0,07 & 2791 & 100 \\
\hline
\end{tabular}

${ }^{\mathrm{a} D o n t} 75$ « humain par extension », c.-à-d. noms collectifs humains, désignations métonymiques, etc. ${ }^{b}$ Dont 237 « humain par extension », c.-à-d. noms collectifs humains, désignations métonymiques, etc.

Tableau 6: Animéité du sujet

juger), ce qui montre que la prédication attributive réalisée par virer véhicule surtout des propriétés associées à des êtres humains (Tableau 6).

Dans ce qui suit, nous allons approfondir et objectiver les différences au moyen d'une variante de l'analyse collexémique standard (4.1), avant de les rapporter au sémantisme du verbe lexical plein (4.2).

\subsection{Divergences : les leçons de l'analyse collexémique distinctive}

Afin d'objectiver au maximum les contrastes entre les deux verbes, nous nous baserons sur une variante de l'analyse collexémique standard, la distinctive collexeme analysis (Gries et Stefanowitsch 2004). Celle-ci permet de comparer les collexèmes de deux constructions synonymes, d'où l'intérêt de la méthode pour l'étude d'alternances du genre He gave Mary the book / He gave the book to Mary. Dans ce cas, les « constructions autres que $\mathrm{C}$ » sont limitées à une seule construction (ou à quelques constructions). En l'occurrence, nous comparerons les attributs de virer et de tourner. En somme, la méthode offre la possibilité de faire un zoom sur les différences, et cela, en dépit du fait que la plupart des classes sémantiques sont partagées par les deux verbes. Il importe de signaler que cette méthode privilégie les items distinctifs; ceux-ci ne figurent donc pas nécessairement dans le top 100 examiné dans le cadre de l'analyse collexémique standard.

Si l'on prend à nouveau le seuil de $p=0.05$ (force d'association >1.30103) comme limite, on constate que le nombre d'items attirés de manière statistiquement significative par l'un des deux verbes est assez réduit (voir le Tableau 7).

Du côté de virer, le regroupement des items attirés par ce verbe (au détriment de tourner) aboutit à trois classes sémantiques : les convictions politiques (écolo, facho/ fasciste, gauchiste, libéral et, par extension, rebelle ${ }^{14}$ ), les maladies mentales (parano(ïaque), dépressif et, par extension, émo(tionnel)) et les couleurs, notamment des nuances de rouge (pivoine, écarlate; puis encore écrevisse $0: 8^{15}$ ).

\footnotetext{
${ }^{14}$ Chiffre dopé par la traduction du titre du livre de Sarah Palin, Going Rogue 'Virer rebelle'.

${ }^{15}$ Les indices des deux côtés du deux-points renvoient aux fréquences absolues du lemme avec tourner et virer.
} 


\begin{tabular}{|c|c|c|c|c|c|c|c|}
\hline \multicolumn{4}{|c|}{ préférence pour tourner } & \multicolumn{4}{|c|}{ préférence pour virer } \\
\hline lemme & $\begin{array}{c}\text { obs.fq } \\
\text { tourner }\end{array}$ & $\begin{array}{l}\text { obs.fq } \\
\text { virer }\end{array}$ & $\begin{array}{c}\text { Force } \\
\text { attraction }\end{array}$ & lemme & $\begin{array}{c}\text { obs.fq } \\
\text { tourner }\end{array}$ & $\begin{array}{c}\text { obs.fq } \\
\text { virer }\end{array}$ & $\begin{array}{c}\text { Force } \\
\text { attraction }\end{array}$ \\
\hline bourrique & 31 & 0 & 192.518 & pivoine & 0 & 24 & 29.074 \\
\hline vinaigre & 41 & 15 & 140.211 & écolo & 0 & 15 & 18.144 \\
\hline aigre & 24 & 2 & 125.837 & facho/fasciste & 2 & 26 & 16.988 \\
\hline chèvre & 28 & 12 & 89.746 & écarlate & 2 & 24 & 15.138 \\
\hline maboul & 16 & 9 & 45.712 & dépressif & 0 & 10 & 12.086 \\
\hline sot & 7 & 0 & 43.139 & gauchiste & 0 & 10 & 12.086 \\
\hline fada & 7 & 1 & 35.137 & libéral & 0 & 10 & 12.086 \\
\hline vert & 17 & 16 & 32.081 & émo(tionnel) & 0 & 9 & 10.875 \\
\hline bleu & 17 & 20 & 25.098 & rebelle & 0 & 9 & 10.875 \\
\hline bizarre & 9 & 6 & 24.817 & parano(ïaque) & 17 & 104 & 2.437 \\
\hline chaud & 4 & 1 & 18.571 & & & & \\
\hline laid & 4 & 1 & 18.571 & & & & \\
\hline négatif & 4 & 1 & 18.571 & & & & \\
\hline mortel & 3 & 0 & 18.465 & & & & \\
\hline vicieux & 3 & 0 & 18.465 & & & & \\
\hline glauque & 6 & 5 & 15.288 & & & & \\
\hline bolchévique & 3 & 1 & 13.314 & & & & \\
\hline cancéreux & 2 & 0 & 12.306 & & & & \\
\hline délirant & 2 & 0 & 12.306 & & & & \\
\hline étrange & 2 & 0 & 12.306 & & & & \\
\hline fadasse & 2 & 0 & 12.306 & & & & \\
\hline haussier & 2 & 0 & 12.306 & & & & \\
\hline nuageux & 2 & 0 & 12.306 & & & & \\
\hline rance & 2 & 0 & 12.306 & & & & \\
\hline sénile & 2 & 0 & 12.306 & & & & \\
\hline sombre & 4 & 3 & 11.956 & & & & \\
\hline jaune & 10 & 17 & 10.204 & & & & \\
\hline dangereux & 6 & 1 & 2.952 & & & & \\
\hline français & 6 & 1 & 2.952 & & & & \\
\hline mauvais & 6 & 2 & 2.45 & & & & \\
\hline vilain & 6 & 2 & 2.45 & & & & \\
\hline noir & 9 & 9 & 1.805 & & & & \\
\hline
\end{tabular}

Tableau 7: Tourner vs virer (analyse collexémique distinctive)

Du côté de tourner ${ }^{16}$, les items attirés se laissent également facilement agglomérer en classes sémantiques, même si le plus souvent les collexèmes sont également largement attestés avec virer:

\footnotetext{
${ }^{16}$ Notons que les chiffres des noms (bourrique, chèvre, vinaigre) sont influencés négativement par la taille plus réduite de l'échantillon tourner + nom (voir la section 1), mais il suffit de se référer aux adjectifs pour voir que les tendances sont évidentes.
} 
- la folie : bourrique (31:0), chèvre, maboul, sot, fada, délirant, sénile. Les lexèmes rattachés au domaine de la folie penchent donc nettement du côté de tourner, même si fou lui-même et la variante familière dingo/gue se répartissent de manière égale sur les deux verbes à tel point qu'ils ne sont attirés par aucun des deux verbes de manière significative (respectivement 168/500; 14/54)

- la dégénérescence

- physique : vinaigre (41:15), aigre (24:2), mortel (3:1), cancéreux (2:0), rance (2:0), par extension (cf. le temps qui se dégrade), nuageux (2:0),

- situation qui dégénère (au figuré) ou qui prend une tournure inquiétante : laid (4:1), négatif (4:1), vicieux (3:0), dangereux (6:1), chaud (4:1, dont trois dans le sens de 'dangereux'), bizarre (9:6), glauque (6:5), vilain (6:2), étrange (2:0);

- les couleurs : vert (17:16), bleu (17:20), jaune (10:17), noir (9:9), sombre (4:3; situation qui dégénère), fadasse (2:0, dont une application à une couleur);

- autres : français (6:1), bolchévique (3:1), haussier (2:0).

Regardons de plus près ces constats. Dans le domaine de ce que nous avons appelé 'la faiblesse mentale', les profils des deux verbes se précisent. S'ils partagent un noyau prototypique (items les plus fréquents et les plus attirés, cf. analyse collexémique simple), les extensions à partir de ce prototype divergent, ce qui donne lieu à une spécialisation divergente. Ainsi, virer penche nettement du côté des maladies mentales (y compris les addictions), alors que tourner s'en tient aux manifestations plus superficielles de la 'folie' prise dans le sens de la 'sottisebêtise', ou encore de l'énervement ou de la rage. Ainsi les locutions figées (faire) tourner chèvre et (faire) tourner (en) bourrique renvoient-elles à un état d'exaspération ou d'abêtissement causé par « des taquineries malveillantes et incessantes » (TLFi, s.v. bourrique) ou des « exigences [ou] taquineries » (Le Grand Robert 2016) ${ }^{17}$, d'après l'image de la chèvre ou de l'âne, deux animaux réputés brusques et connaissant des accès de violence soudaine (cf. aussi cabriole, caprices, etc.).

Le deuxième domaine, celui de la dégénérescence (domaine sans doute le plus emblématique de tourner), ne semble pas avoir de pendant du côté de virer (v. section 3.1 , analyse collexémique simple $)^{18}$. La dégénérescence est présente dans les cooccurents qui rappellent le sens péjoratif de tourner intransitif (le lait qui tourne, v. la section 4.2). Cet héritage est encore nettement présent dans les collexèmes les plus attirés par tourner au détriment de virer : vinaigre (41:15), aigre (24:2), rance (2:0). Cet emploi a connu aussi des extensions dans le domaine de la maladie [mortel (3:1), cancéreux (2:0)], de la météo [nuageux (2:0), emploi appuyé sans doute par l'expression tourner à l'orage; notons que le temps tourne

\footnotetext{
${ }^{17}$ Les dictionnaires mentionnent aussi rendre chèvre et la locution vieillie prendre la chèvre (TLFi).

${ }^{18}$ Le Grand Robert (s.v. virer) observe toutefois que l'emploi intransitif de virer pour marquer le changement de coloris peut également être associé à un processus de dégénérescence : « changer d'aspect, voir ses caractéristiques s'altérer (en parlant de substances, de coloris), p. ex. un maquillage qui vire, un parfum trop vieux qui a viré (changement d'odeur et de coloris) ».
} 
au vinaigre est également attesté] et dans le domaine des rapports intersubjectifs ou, de manière plus générale, les situations intersubjectives, comme le montre de manière éloquente l'adjectif aigre (24:2) dont un tiers des occurrences concernent le sens physique, deux tiers le sens métaphorique 'désagréable' (rapports, discussions, idylles... qui tournent aigres). D’autres exemples sont vilain (6:2; avec des sujets inanimés : ça, le jeu, etc. 'dangereux et déplaisant', Petit Robert) et laid (4:1; cf. une bataille qui tourne laide), glauque (6:5), mauvais (6:2; prendre un tournant dangereux, y compris en parlant d'une personne, cf. aussi la catégorie des types humains 'détestables'), négatif (4:1; en parlant d'un index boursier censé prédire l'évolution de l'économie), dangereux (6:1), vicieux (3:0) et chaud ('dangereux' dans 3 des 4 exemples). Parmi les collexèmes attirés de manière non significative, on trouve malsain (2:1), violent (3:3), méchant (6:9), sauvage (2:1), âcre (1:0) et calamiteux (1:0) qui viennent compléter la série, et parmi les collexèmes significatifs, par extension, bizarre (9:6) et étrange (2:0). Notons qu'avec des items comme vinaigre et aigre, l'expression devient moins transparente (compositionnelle), même si l'on peut encore reconnaitre deux composants sémantiques qui maintiennent une certaine compositionnalité (cf. les idiomatically combining expressions de Nunberg et al. 1994). Il s'ensuit parfois des extensions analogiques créatives mettant en œuvre des mots qui s'alignent exceptionnellement sur un autre paradigme sous la pression de la construction (v. Audring et Booij 2016; Er geht mir auf DET N 'il me tape sur les (nerfs)') comme tourner cacahuète, expression par analogie avec partir en cacahuète 'prendre un mauvais tournant' 19 .

Reste la troisième grande catégorie, les couleurs. Curieusement, tourner partage les couleurs avec virer, mais on note cependant une certaine spécialisation. Tout d'abord, virer se combine souvent avec des tons de rouge (rouge lui-même étant partagé : $34: 124)$ : pivoine $(0: 24$ + couleur pivoine $0: 3)$, écarlate $(2: 24)$ et, parmi les collexèmes non significatifs, écrevisse $(0: 8)$, roux $(0: 5)$, tomate $(0: 4)$, tabac $(0: 3)$, couleur rouille $(0: 2)$, cramoisi $(1: 3)$ et orange (3:15). Il semble que l'attraction des tons de rouge s'explique en grande partie par leur rôle dans le domaine des changements faciaux : quelqu'un qui vire \{pivoine / écrevisse / écarlate / tomate devient tout rouge à cause d'un sentiment d'embarras ou à la suite d'une insolation, ce qui fait en partie le lien avec la sphère mentale, l'un des terrains de prédilection de virer. En outre, virer s'utilise souvent pour marquer un changement au niveau de la teinte des cheveux (virer blond [0:5], poivre et sel [0:5], etc.). Tourner, de son côté, attire davantage les changements de couleur considérés comme néfastes : noir (9:9), sombre (4:3), fadasse (un exemple concernant le coloris), ou encore pâle (2:3), gris (4:9), terne (1:0), incolore (1:0), semi-bleu (1:0) et, de manière plus spectaculaire, le groupe des adjectifs de couleur péjoratifs en -âtre : jaunâtre (2:1), grisâtre (2:1), bleuâtre (1:0), brunâtre (1:1), rougeâtre (1:1), verdâtre (1:1). Ces couleurs confirment donc ce qu'on pourrait appeler le caractère encore plus sinistre de tourner par rapport à virer.

Il reste à commenter les quelques types lexicaux isolés. Tout d'abord tourner français, qui remonte à une expression vieillie signifiant 'se convertir, se rallier au

\footnotetext{
${ }^{19}<$ https://fr.wiktionary.org/wiki/partir_en_cacahou\%C3\%A8te> (consulté le 20/05/2016).
} 
camp adverse', pendant intransitif d'un emploi transitif (v. Van Wettere et Lauwers 2017), nuance qui survit également dans l'expression tourner casaque. On peut y rattacher bolchévique qui, à travers l'association avec tourner (3:1), se rapproche de la trahison, comme dans l'exemple (26) (voir aussi l'expression tourner haussier, qui a été commentée à la section 3.2.2).

(26) Voilà qu'il nous trahit, voilà qu'il tourne bolchévique [il s'agit de Sarkozy]

(Sketch Engine)

\subsection{Bilan : la spécialisation sémantique des deux verbes attributifs face à leur profil sémantique général}

Après avoir distingué les profils sémantiques de virer et tourner ${ }^{20}$, il convient de reprendre un peu de hauteur et de mettre en rapport les asymétries relevées dans les emplois attributifs - c'est-à-dire des emplois comme outils grammaticaux donc auxiliarisés et grammaticalisés - avec le sémantisme des emplois pleinement lexicaux des deux verbes. Cette démarche nous permettra de formuler quelques hypothèses sur la persistance lexicale (lexical persistence, Hopper 1991), c'est-à-dire les contraintes distributionnelles qui rappellent, à un niveau certes plus abstrait, certaines caractéristiques de la sémantique du verbe lexical.

En somme, l'analyse collexémique distinctive a montré que le caractère défavorable du changement d'état exprimé par le verbe attributif est encore plus prononcé avec tourner, comme le montre sa prédilection pour la dégénérescence physique et intersubjective et les couleurs 'néfastes'. D'autre part, s'agissant de débilité mentale, le caractère défavorable de tourner est affaibli par le fait que tourner n'attribue pas la 'folie' (ou la sottise) à un état maladif de l'esprit, contrairement à virer, même si, là encore, on observe souvent une banalisation du sens des cooccurrents (faut pas virer fou, virer parano, etc.). L'attraction de virer pour le mental apparait aussi clairement dans le domaine des convictions idéologiques et sera confirmée par l'analyse des cooccurrents de la nuance 'inclination' (v. section 5).

Comment ces différences se rapportent-elles au sens de base du verbe lexical? Pour tourner, nous avons déjà mis en évidence les liens étroits que l'on peut établir entre les emplois attributifs et les emplois intransitifs pour ce qui est de la putréfaction $^{21}$. L'origine de cette nuance semble remonter à l'emploi intransitif du verbe tourner avec un sujet dénotant une substance organique susceptible de s'altérer (du lait, de la sauce, des fruits, etc.) et/ou de 's'aigrir' ou de 's'acidifier' (du vin). D'où également les combinaisons le vin tourne à l'aigre / au vinaigre. Voilà donc une « évolution interne, c'est-à-dire non liée à une cause extérieure, mais en quelque sorte déjà «programmée » par la nature de $\mathrm{N}_{0}$ : il est dans la nature du lait d'avoir une certaine évolution et de devenir aigre », comme l'affirme Leeman (2004 : 44) à propos de tourner à / en.

\footnotetext{
${ }^{20}$ Notons que la différence entre les deux verbes n'est pas à chercher dans le domaine aspectuel, car les deux verbes s'accompagnent de compléments et d'auxiliaires aspectuels exprimant tantôt le déroulement (en train de), tantôt la phase inchoative (commencer à).

${ }^{21}$ Leeman (2004) attribue le caractère péjoratif de tourner à à l'imprévisibilité (cf. aussi le temps tourne au beau).
} 
Le deuxième pont que l'on peut établir entre les emplois attributifs et les emplois intransitifs concerne les manifestations plus superficielles de la 'folie', de l'énervement ou de la rage (tourner \{chèvre / bourrique\}). On peut y voir une extension métaphorique de la rotation d'un être autour de son axe qui peut causer le tournis (la tête me tourne), ou encore, des va-et-vient causant l'énervement (tourner dans sa cage), et donc la perte de son discernement, notamment en parlant d'animaux réputés têtus comme l'âne ou la chèvre. On peut supposer que la rotation 'débridée' et incontrôlée de la vis qui tourne fou ou de la machine qui ne tourne pas rond a également alimenté cette dimension sémantique, d'autant plus que les frontières entre les domaines adverbial et attributif y sont extrêmement ténues.

Qu'en est-il pour virer? Ses emplois marquant des changements de couleur remontent à un emploi intransitif, qui a aussi un pendant transitif (virer une image 'la faire changer de couleur'). L'attribut du sujet, facultatif à l'origine, y est devenu essentiel par la suite. De même pour virer positif : une cuti(-réaction) qui vire devient positive par l'apparition d'une large rougeur provoquée par la tuberculine; l'adjectif positif est lié à la couleur par métonymie (v. Van Wettere et Lauwers 2017).

Reste le second noyau sémique du verbe, celui associé aux convictions (politiques, mais aussi alimentaires, religieuses, etc.) et aux maladies mentales. Comment celui-ci serait-il lié aux autres emplois - lexicaux - du verbe? La réponse est moins évidente, mais nous tenterons l'hypothèse selon laquelle l'origine remonte à certaines particularités des emplois spatiaux du verbe, notamment dans le domaine de la navigation à voile, comme le montre l'expression virer (de bord) 'changer de direction pour un navire en prenant le vent du côté opposé à celui d'où il venait' (TLFi). Cette définition recèle deux sèmes qui s'avèrent cruciaux pour expliquer les effets de sens décrits ci-dessus. Ainsi, au sème 'changement de direction' (que le verbe partage avec tourner) s'ajoute la nuance de 'direction opposée' (cf. aussi un revirement de la situation) - en réalité l'angle est de $90^{\circ}$ - et surtout celle de changement d'amure. Le changement d'amure réfère au fait que le côté du bateau qui reçoit le vent change au cours d'une manœuvre, illustré dans la Figure 1.

Formulé de manière plus abstraite, on assiste donc à un passage de $\mathrm{A}$ à $\mathrm{B}, \mathrm{A}$ et $\mathrm{B}$ étant deux positions discrètes. Le deuxième élément crucial dans la définition de virer (de bord) est que le changement de direction 'bipolaire' s'effectue en fonction du vent. On comprend dès lors que la métaphore de l'aisance (opportuniste...) avec laquelle on change d'opinion est dans l'air, comme le montrent non seulement le sens métaphorique de virer de bord mais aussi l'expression virer (ou tourner) à tout vent 'changer facilement d'avis, être influençable', ou encore, virer sa cuti 'changer brusquement de conviction' et prendre un virage en politique ${ }^{22}$. Nous croyons retrouver dans les emplois attributifs de virer le caractère bipolaire du changement de direction. Il en résulte aussi un changement souvent brusque, mais pas nécessairement. Le caractère 'bipolaire' du changement apparait aussi dans une série d'indices distributionnels :

${ }^{22}$ La versatilité en politique est également couverte par (re)tourner : tourner casaque, retourner sa veste. 


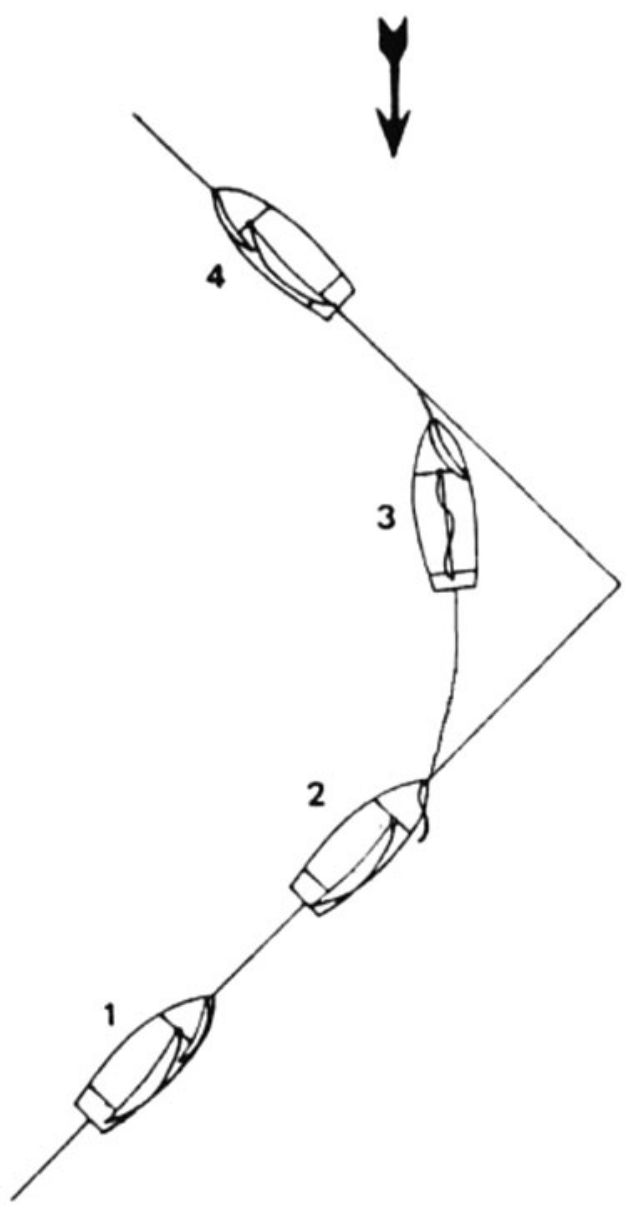

Figure 1: Virer de bord

(i) Une construction morphosyntaxique spécifique : le changement de $\mathrm{A}$ à $\mathrm{B}$ est parfois marqué par $d e$... (à). Cette construction est très courante pour la construction indirecte virer à exemple (27), mais peut se maintenir dans la construction directe, à l'instar du verbe devenir, comme en (28).

(27) Mais que celles qui virent facile $d u$ grand bonheur aux larmes

(Sketch Engine)

(28) a. de ... X, il est devenu $Y$

(Melis 2007 : 232-233))

b. qui de païen a viré ultra catho

(Sketch Engine)

La même construction est également attestée avec tourner à, mais s'avère beaucoup plus marginale. Ainsi, dans un échantillon de chaque fois 15000 exemples (recherche lexicale virer / tourner), on trouve respectivement 81 exemples de virer de... à contre seulement 3 exemples de tourner de... à. 
(29) De septembre à novembre, le ton du vert des olives évolue, les olives tournent $d u$ vert plus soutenu au violet puis au noir

(Sketch Engine)

(ii) La présence de davantage d'adjectifs attributs au sémantisme discret. Rappelons à ce propos les trois classes sémantiques distinctives pour virer : les convictions politiques, les maladies mentales (parano(ïaque), dépressif) et les couleurs, notamment les nuances de rouge. On pourrait y ajouter les noms dénotant des orientations sexuelles (gay, lesbienne, pédé, homo(sexuel)) qui se retrouvent dans les collexèmes de virer donnés en (18). Notons que ces adjectifs (ou ces noms, si on préfère cette analyse) ne sont normalement pas gradables, pas plus que les adjectifs de couleur, que l'on sait également difficilement gradables. En plus de la discrétude - on est communiste ou on ne l'est pas -, ces items sont souvent associés à une certaine bipolarité : on est de gauche ou de droite, homo ou hétéro, malade mental ou en parfaite santé mentale.

(iii) Sur le plan morphologique, on note la présence des préfixes bipolaires anti/pro avec virer, que l'on ne trouve que marginalement avec tourner:

- anti : anti-sémite, anti-capitaliste, anti-héros, anti-impérialiste, antichallenge, antichrist, antinucléaire, antisioniste, anti-Choi, anti-jeunes, anti-juif, anti-national

- pro : pro-Sarko, pro-Bayrou, pro-communiste, pro-Ségo, pro-sioniste

Le changement bipolaire jette une lumière (transversale) toute nouvelle sur les cooccurrents de virer. Cette dimension du changement semble plus ou moins absente dans le sémantisme de tourner attributif.

\section{TOURNER / VIRER : CARACTÉRISATION DE LA CONSTRUCTION DIRECTIONNELLE ABSTRAITE EXPRIMANT UNE INCLINATION OU UNE ORIENTATION}

Dans ce qui précède, nous avons contrasté les profils des deux verbes attributifs, tout en rattachant les nuances spécifiques dont ils témoignent à d'autres éléments du sens lexical. Jusqu'ici nous avons fait abstraction de l'emploi étiqueté 'inclination/orientation', exemplifié en (8), repris ici en (30), et correspondant à 5,5\% des occurrences attributives des deux verbes (cf. Tableau 1).

(30) cette année j'ai viré manga

(Sketch Engine)

Comme nous le verrons, il s'agit d'une construction au profil morphosyntaxique différent, à tel point que l'on pourrait parler de constructions homonymiques (en surface, du moins). Pour le mettre en évidence, nous allons dans un premier temps, à partir de propriétés (morpho)-syntaxiques, distinguer la construction directionnelle abstraite de la construction proprement attributive exprimant le changement d'état ('devenir') (section 5.1). Puis, nous étudierons les profils morphosyntaxique, distributionnel et sémantique de virer et tourner (section 5.2).

\section{1 'Inclination / orientation' vs 'changement d'état': quelle différence?}

La première différence apparait lorsque l'on cherche à remplacer tourner/virer par devenir : les emplois directionnels abstraits sont rétifs à la commutation avec 
devenir (et être), pourtant deux copules réputées peu sélectives (cf. Lamiroy et Melis 2005, Lauwers et Tobback 2010), ce qui pointe vers un moindre degré de copularité. Certes, la copule être peut exprimer l'inclination, mais pour cela elle doit entrer dans la locution [être + adverbe de degré + nom, p. ex. je suis très fromage], comme Lauwers (2014) l'a montré, ce qui n'est nullement le cas de virer/tourner 'inclination', qui n'acceptent même pas la modification de degré, comme on voit en (31).

(31) a. J'ai viré death metal.

b. ?? Je suis death metal.

c. Je suis assez death metal. ('J'aime assez le death metal')

d. * J'ai viré très death metal.

Une autre différence s'observe du point de vue de la catégorie morphosyntaxique des cooccurrents attributifs. La présence d'adjectifs relationnels (non gradables, donc) est pour le moins curieuse étant donné la difficulté avec laquelle les adjectifs relationnels s'emploient en position d'attribut (voir cependant Nowakowska 2004). Qui plus est, ces adjectifs relationnels ne s'accordent pas avec les noms auxquels ils se rapportent, comme on le voit en (32) et (33).

(32) [...], empêchent les autres de virer bio.

(Sketch Engine)

(33) j'rappelle quand même que la plupart des grandes villes possédant des ghettos sont démocrates, et leurs dirigeants sont trés tournés social

(<http://www.allocine.fr/communaute/forum/message_gen_nofil=487286\&cfilm=\&ref personne $=\&$ carticle $=\&$ refserie $=92 \&$ refmedia $=$. html $>$ )

Voici encore quelques exemples avec virer : illégal ('dans l'illégal'), [jazz qui vire] éléctrique, pluriculturel total, hispanique, tzigane; et avec tourner: multijoueur, social, [jazz]funky, comique/burlesque, international, acoustique, aérien, argentique, chic et urbain, outre-Atlantique, pervers et conservateur. Ces exemples peuvent tous être glosés par 'orienté sur le domaine ADJ'. Comme il est difficile de postuler à chaque fois la présence d'une tête nominale ellipsée - fût-ce une ellipse mémorielle comme dans la (ville) capitale - il vaut mieux analyser ces emplois comme des adjectifs relationnels substantivés désignant un domaine ou un genre : le bio, l'équitable, l'international, le 'multijoueur'. La substantivation explique l'invariabilité, à l'instar de la substantivisation appelée « neutre » d'adjectifs en noms abstraits (important > l'important; cf. le neutre en espagnol ou en allemand : importante > lo importante; wichtig > das Wichtige; cf. Lauwers 2008). Lorsqu'il existe un homonyme pleinement nominal, comme technique en (34), on peut d'ailleurs hésiter quant à l'analyse : s'agit-il de l'adjectif substantivé (le technique 'tout ce qui est technique') ou du substantif (la technique)?

(34) À cette date, il n'y a pas de service Emballage et le poste acheteur est très tourné technique. (Google)

Quelle que soit l'analyse que l'on propose pour ces adjectifs invariables, il convient de ne pas les confondre avec les adjectifs (parfois homonymes) qualificatifs exprimant l'état résultant ('devenir ADJ'). Ainsi, dans (35), très tourné pervers veut dire 'très attiré par tout ce qui est pervers', et non pas 'devenu pervers' (il tourne pervers). 
(35) Je savais que j'étais très tourné pervers, mais là...

(Sketch Engine)

Certes, certains adjectifs a priori non gradables tendent à se comporter comme des adjectifs qualificatifs, mettant en oeuvre une échelle qui facilite tant l'insertion d'adverbes de degré (sans glissement de sens) que la commutation de virer/tourner par devenir (cf. un blog qui vire porno, une marque qui vire commerciale, etc.).

(36) le débat qui ne manquerait pas de virer politique

(Sketch Engine)

On ne saurait pas non plus confondre ${ }^{23}$ les adjectifs relationnels avec les formes ambivalentes (adjectifs et noms) qui dénotent une tendance idéologique (gauchiste, fasciste, communiste, etc.; musulman, etc.; goth, punk, etc.) présentée comme le résultat d'un changement d'état (' $\mathrm{X}$ est devenu ADJ/N').

(37) Sarkozy n'a pas viré anti-impérialiste et son atlantisme ne s'arrête pas au bas d'un contrat à 7 ou 8 chiffres

(Sketch Engine)

Que les adjectifs que l'on trouve derrière les deux verbes s'avèrent être des adjectifs substantivés ne devrait pas trop nous étonner, finalement, car la position postverbale présente une orientation nettement nominale, comme le montrent les chiffres du Tableau 8.

Ce profil plutôt nominal constitue une autre différence avec le profil général des deux verbes. Ils ont en effet une préférence pour les adjectifs (chi carré 34.745, $d f=1$; $p=3.758 \mathrm{e}-09$ ), comme on le voit dans le Tableau 9.

Ces préférences pour le domaine nominal correspondent aux exigences du sens : l'expression d'une orientation/inclination requiert normalement une expression nominale référant à l'entité sur laquelle est orienté le support ou base de la prédication, comme dans (8) et (38).

(38) on tourne extrême-droite dès 30 ans

(Sketch Engine)

Cette orientation/inclination peut se rapporter à un secteur de l'échiquier politique, à un genre musical (et, par extension, artistique), à une conviction religieuse, à un genre vestimentaire ou, notamment avec virer, à n'importe quelle 'idéologie' informatique/alimentaire/etc., parfois liée à la préférence pour une marque spécifique comme dans (39) et (40).

(39) Tout au long de l'enfance, c'est dentelle rose Vs plastique noir. A l'adolescence, quand tout le monde vire black ou fluo kids, il n'y a que des perdants. (Sketch Engine)

(40) maintenant 1 an et demi que je suis viré Mac

(Sketch Engine)

Si l'idée d'une inclination/orientation demande en principe un agent humain, celui-ci peut être impliqué par voie métonymique dans un support inanimé (la poste $>$ les dirigeants de la poste), comme en (41).

\footnotetext{
${ }^{23}$ Certes, les liens avec le domaine nominal sont étroits, car sous l'adjectif-nom exprimant la caractérisation du référent sujet se cache une relation orientée vers une entité nominale, comme il ressort clairement de la paire suivante : a. les ouvriers et les fonctionnaires pensent à virer sarkozy ('tendent à voter Sarkozy') <http://www.utpjournals.com/blog/wpcontent/uploads/2011/11/CJL-Style-Guide.pdf> vs b. virer sarkozyste ('devenir sarkozyste') (Sketch Engine).
} 


\begin{tabular}{llll}
\hline \hline & ADJ & $\mathrm{N}$ & TOTAL \\
\hline Tourner & $34,7 \%(17)$ & $65,3 \%(32)$ & $100 \%(49)$ \\
Virer & $14,3 \%(22)$ & $85,8 \%(132)$ & $100 \%(154)$ \\
\hline \hline
\end{tabular}

Tableau 8: Catégories morphosyntaxiques de la construction directionnelle abstraite

\begin{tabular}{lrrr}
\hline \hline \multicolumn{2}{c}{ ADJ } & $\mathrm{N}$ & \multicolumn{2}{l}{ TOTAL } \\
\hline $\begin{array}{l}\text { Virer } \\
\text { Tourner }\end{array}$ & $689[<624](43,5 \%)$ & $841^{24}[<270](\mathbf{5 6 , 5} \%)$ & $1531[<894](100 \%)$ \\
\hline \hline
\end{tabular}

Tableau 9: Recherche étiquetée dans le French Ten Ten corpus

(Sketch Engine)

(41) La poste est privatisée de fait, et a viré marketing

(Sketch Engine)

Notons que cet effet inclination/orientation qui demande deux entités conceptuellement distinctes (correspondant à deux participants) disparait à chaque fois qu'un genre est prédiqué d'un autre genre qui, de ce fait, fait l'objet d'une métamorphose ('devenir'); le nouvel état devient consubstantiel avec l'entité exprimée par le sujet, pour utiliser la terminologie ancienne, ou encore, la dichodèse devient syndèse, pour le dire avec les mots de Damourette et Pichon (1930-1949) (v. Lauwers $2004: 318-322$ ).

(42) Son death vire power-trash, parfois limite hardcore

(Sketch Engine)

Autre différence qui découle de la relation inclination/orientation entre un sujet humain et un autre référent, c'est l'absence de co-variation en genre et en nombre. Cette absence s'observe en (43); le nombre du nom sujet est indépendant du nombre du nom attribut (v. Lauwers 2014) :

(43) Pierre (singulier) est très fruits exotiques (pluriel)

Or, dès que la construction passe de l'inclination (impliquant deux entités) au changement d'état (impliquant une seule entité, le référent sujet), on constate non seulement que la commutation avec devenir devient possible mais que l'accord semble lui aussi s'imposer en français contrôlé, comme dans (44) et (45).

(44) L'Allemagne vire végétarienne

(Sketch Engine)

(45) La femme à talleyrand aurait pu aussi virer nazie

(Sketch Engine)

En soi, l'orientation nominale éloigne la construction directionnelle du prototype du verbe attributif qui, lui, préfère l'adjectif qualificatif (Hengeveld 1992), catégorie

\footnotetext{
${ }^{24}$ Les chiffres en italiques sont obtenus par extrapolation à partir d'un échantillon randomisé, tout en ajustant l'extrapolation pour les erreurs d'étiquetage commises par Sketch Engine.
} 
prédicative, c'est-à-dire non référentielle. Dans le cas des constructions directionnelles, la nature référentielle est d'ailleurs encore plus prononcée, comme en témoigne, dans (46), la reprise avec un pronom démonstratif qui semble encore envisageable, ou en tout cas meilleure qu'avec être.

(46) a. Les petits commerçants ont (massivement) viré Sarkozy. Celui-ci $i_{i}$ leur avait donné plein de cadeaux fiscaux.

b. Ce spectacle était tourné jeune public ${ }_{i}$. Celui-ci $i_{i}$ lui a fait bon accueil.

c. Pierre est devenu un excellent professeur ${ }_{i}$. ${ }^{*}$ Celui-ci $i_{i}$ est toujours applaudi à la fin de ses cours.

Dernière différence, la construction directionnelle abstraite présente moins de propriétés attributives, à tel point que l'on peut mettre en doute son statut attributif. Or, si ce n'est pas un attribut, qu'est-ce que c'est alors? La réponse impose un détour par le domaine adverbial. En effet, la construction directionnelle abstraite reste très proche des constructions à préposition directionnelle (vers, sur, du côté de/côté), comme on le voit en (47) et (48), dont elle semble être une espèce de raccourci.

(47) un staff médical très tourné vers l'international $<$ www.cclj.be/actu/politique.../belges-et-israeliensmain-dans-main> consulté le 05/11/2017

(48) Rock qui “vire vers l'électro" (18 attestations sur Google, consulté le 21/06/2016)

On ne saurait cependant y voir le résultat d'une simple ellipse. On note, en effet, des rajustements concomitants dus à l'effacement de l'article. Ainsi, l'on sait que les noms nus n'ont pas le même potentiel de modification que les noms déterminés (Lauwers 2011 et l'aperçu de la littérature fourni dans cet article). De manière générale, ils n'acceptent que des modificateurs indiquant des sous-types de l'entité dénotée par le nom en question, ce qui correspond à l'effet généralisant qu'ils induisent. Pour ce qui est de ces rajustements liés à l'absence de l'article, un parallèle peut être établi avec la construction dans (49), que nous avons déjà citée et qui se comporte comme une locution verbale attributive dans laquelle l'adverbe de degré modifie l'ensemble $<\mathrm{V}+$ prédicat nominal $>$ (Lauwers 2014) ${ }^{25}$.

(49) Je suis plutôt $\{$ fromage/café/... $\}$

Les emplois directionnels abstraits de virer et tourner seraient donc des constructions sui generis, proches de la construction à SPrép directionnel abstrait, que l'on ne saurait prendre pour des constructions attributives. Pour élucider plus avant ce mystère, il faudrait comparer minutieusement les cooccurrents avec ceux de virer/tourner à, etc. et regarder en détail les autres contextes dans lesquels une préposition (directionnelle) peut disparaître ${ }^{26}$, ce qui n'entre plus dans notre propos ici.

\footnotetext{
${ }^{25}$ Qui n'est pas possible, cependant, avec virer, mais attesté avec tourné participe (voir l'exemple (57) ci-dessous).

${ }^{26}$ Dans le cas de tourner, la construction participiale pourrait avoir servi de construction de transition, à l'instar des synonymes porté sur et orienté sur : Il est (très) porté sur les plaisirs sexuels (Google) > un événement (très) porté business (Google).
} 


\begin{tabular}{|c|c|c|c|c|c|c|c|}
\hline & \multicolumn{4}{|c|}{ Participe passé } & \multirow{2}{*}{\multicolumn{2}{|c|}{$\begin{array}{l}\text { Autres emplois } \\
\text { autres emplois }\end{array}$}} & \multirow[t]{3}{*}{ TOTAL } \\
\hline & \multicolumn{2}{|c|}{$\begin{array}{l}\text { épithète, apposition, } \\
\text { attribut de l'objet }\end{array}$} & \multicolumn{2}{|c|}{ être + participe } & & & \\
\hline & 'devenir' & inclination & 'devenir' & inclination & 'devenir' & inclination & \\
\hline Tourner & 6 & 27 & 4 & 11 & 835 & 11 & 894 \\
\hline Virer & 20 & 0 & 34 & 1 & 2583 & 153 & 2791 \\
\hline
\end{tabular}

Tableau 10: Forme du verbe (participe passé ou autre) vs sens

\subsection{Tourner vs virer directionnels : divergences}

Si les emplois directionnels des deux verbes partagent des caractéristiques (morpho)syntaxiques et sémantiques qui les distinguent des emplois proprement attributifs (section 5.1), il n'en reste pas moins que les profils de virer et tourner ne sont pas identiques.

\subsubsection{Des profils morphosyntaxiques différents}

Sur le plan morphosyntaxique, on note une différence majeure entre les deux verbes pour ce qui est de la manière dont ils s'intègrent dans la phrase lorsqu'ils sont utilisés en emploi directionnel. Ainsi, pour tourner la nuance inclination/orientation est étroitement corrélée à l'emploi de la forme participiale (cf. Tableau 10), en épithète (et en apposition) (50), en attribut de l'objet (51), ou en combinaison avec l'auxiliaire être (52).

(50) le contenu du programme tourné innovation-nouvelles technologies

(51) $[\ldots]$ dispose de composants à la pointe et se veut tourné multimédia

(Sketch Engine)

(52) tu es assez tourné international à ce que je vois!

(Sketch Engine)

(Sketch Engine)

Notons que virer se combine également avec être, mais c'est toujours en emploi attributif marquant un changement d'état, comme en (53).

(53) le fait qu'il est viré gay peut avoir un avantage

(Sketch Engine)

Nous n'avons trouvé qu'un seul contre-exemple, c'est-à-dire la phrase en (54).

(54) Cela va faire maintenant 1 an et demi que je suis viré Mac

(Sketch Engine)

Pour tourner, jusqu'à 81,8 \% (27/33) des exemples en emploi épithète/apposition/ attribut de l'objet expriment l'inclination/l'orientation. Il en est de même de 73,3 $\%$ des occurrences construites avec l'auxiliaire être. Inversement, on constate que $77,5 \%$ des emplois (38 des 49) de tourner de type 'inclination' proviennent des emplois comme épithète/apposition/attribut de l'objet ou en combinaison avec être. Autrement dit, pour tourner, il y a une très forte corrélation entre la nuance 'inclination/orientation' et le statut plus adjectival du participe (chi carré 531.615; $p=0 ; d f$ $=1$ ), mais celle-ci n'est pas exclusive : on trouve aussi des exemples en dehors des 
deux constructions 'adjectivales' (55) et les constructions 'adjectivales' (56) autorisent aussi des interprétations en dehors de la sphère de l'inclination/orientation.

(55) $[\ldots]$ de loin mes préférés, avant qu'ils ne tournent électro

(Sketch Engine)

(56) à leur place viennent ancien Patriot Scott Pioli et ancien Cardinal Coordonnateur offensif tourné entraîneur-chef Todd Haley respectivement

(Sketch Engine)

De plus, on constate que la forme participiale de tourner connaît des emplois adjectivés (lexicalisés) dans lesquels il devient gradable par un adverbe de degré; même la coordination avec un adjectif à part entière devient possible.

(57) Eh ben dis donc l'article est trèèès tourné conservateur et limite xénophobe...

(Sketch Engine)

Sur ce point tourner se distingue de virer, qui ne semble pas connaître un tel emploi adjectivé $^{27}$.

Pour ce qui est des cooccurrents à droite du verbe, l'on note une représentation plus forte des adjectifs relationnels substantivés pour virer (section 5.1).

\subsubsection{Profils distributionnels : tourner (orientation) vs virer (inclination)}

Les deux verbes se distinguent également par la sémantique de leurs cooccurrents, à la fois du côté du sujet et du constituant postverbal.

Examinons d'abord le constituant postverbal. Notons que nous passons ici d'emblée du niveau du collexème à celui des classes sémantiques, étant donné que les fréquences des types sont très basses (Tableau 11).

La principale différence entre tourner et virer peut être résumée ainsi : virer marque un changement d'inclination, alors que tourner exprime plutôt un changement d'orientation. Tourner peut se construire avec un $\mathrm{N}$ dénotant une 'cible' vers laquelle tend un support inanimé : 'X est tourné vers le/la N, axé sur le/la N', que N soit un public-cible, un secteur d'activités ou autre chose encore.

(58) Film/spectacle tourné jeune public; système tourné politique de collecte; jeu tourné action; une connaissance tournée pratique

(Sketch Engine)

Dans ces exemples, le sens directionnel ('orienté sur') est encore très présent, ce qui fait que virer y est moins à sa place. On notera d'ailleurs deux attestations de outre-Atlantique - une orientation géographique - parmi les cooccurrents de tourner.

L'analyse des sujets nous permet de préciser le contraste entre les deux verbes. Virer est davantage lié à l'expression d'une (nouvelle) inclination liée à un support humain, comme le montrent les 121 sujets humains contre seulement 32 inanimés (1 ex. indécis), conformément au profil global du verbe, d'où des préférences musicales (ou plus généralement artistiques), politiques et vestimentaires. Tourner, de son côté, compte 16 sujets humains contre 25 sujets inanimés.

${ }^{27}$ Même si l'analogie avec tourner semble parfois donner lieu à des occurrences isolées qui ne sont guère acceptables pour un locuteur natif : Le dernier titre (Bloons Monkey city) est très viré communautaire <http://culturegeek.probb.fr/t102-jeu-flash-la-serie-des-bloons-td> Mon ler mari était très "viré" sur le sexe, j'ai peut êre [sic] été la plus cocufiée de France $<\mathrm{http}: / / \mathrm{www}$.atoute.org/n/forum/showthread.php?t=81606>. 


\begin{tabular}{lcc}
\hline \hline & Virer & Tourner \\
\hline genre musical (pop, rap, ...) & $61,7 \%(95)$ & $30,6 \%(15)$ \\
tendance politique (droite, sarko, modem, ...) & $13,0 \%(20)$ & $6,1 \%(3)$ \\
genre vestimentaire (ou d'équipement) $($ fashion, casual, ...) & $4,5 \%\left(7^{\mathrm{a}}\right)$ & $2,0 \%(1)$ \\
genre artistique (science-fiction, manga, bal-musette, ...) & $4,5 \%(7)$ & $4,1 \%(2)$ \\
objet préférentiel (langue, Mac, scooter, ...) & $3,2 \%(5)$ & $2,0 \%(1)$ \\
système religieux (façon charia, ...) & $1,9 \%(3)$ & $0,0 \%(0)$ \\
cible (marketing, informatique, multimédias, ...) & $11,0 \%(17)$ & $55,1 \%(27)$ \\
\hline TOTAL & $100 \%(154)$ & $100 \%(49)$ \\
\hline \hline
\end{tabular}

${ }^{\mathrm{a}}$ Dont 1 exemple lié à une couleur: virer black ou fluo kids

Tableau 11: Classes sémantiques des cooccurrents (construction directionnelle abstraite)

Enfin, la notion de cible nous permettra de révéler un autre contraste. En effet, si tourner se combine plus souvent avec une cible, on ne saurait cependant nier que même virer accepte cet emploi : virer \{analogue, graphique, marketing, bio, commercial, illégal, solaire $\}$. Toutefois, avec virer ${ }^{28}$, il s'agit non seulement d'un changement d'orientation mais aussi et surtout d'un changement de point de vue (subjectif), de conviction, ce qui a bien sûr partie liée avec le support typiquement humain de virer. Aussi, si une entreprise vire marketing elle 'goes all marketing', ce qui n'est pas la même chose que tourner marketing (et être tourné marketing). De même pour virer bio ou virer solaire. Dans le premier cas, virer marque un changement de conviction 'stratégique', voire même d'idéologie (voir les autres emplois de virer dans la section 3), de politique d'entreprise, alors que tourner marque un simple changement d'orientation. En d'autres termes, virer exprime un changement au niveau de la caractérisation interne ${ }^{29}$ (intériorisé, en profondeur) ${ }^{30}$, alors que tourner dénote une caractérisation plus externe, plus superficielle, qui n'atteint pas 1' 'ADN' du support. L'idée de 'choix idéologique' explique aussi pourquoi virer se combine parfois avec des objets symboliques, porteurs d'une certaine

\footnotetext{
${ }^{28}$ En outre, virer s'utilise pour marquer un changement de sujet de la conversation. Cet emploi n'est pas attesté dans le corpus Sketch Engine pour tourner (une discussion qui vire famillelécologie/etc.), mais ne semble pas impossible : ça parle un peu plus loin et ça tourne écologie <www.paperblog.fr > talents > journal intime $>$.

${ }^{29}$ Nous remercions le lecteur qui nous a permis de préciser et d'éclairer ce contraste.

${ }^{30}$ Certes, avec des supports humains, tourner déclenche souvent une implicature conversationnelle qui le rapproche de virer : être orienté (tourné) sur un domaine implique souvent que l'on ait fait le choix (idéologique) de ce domaine. Ainsi, un esprit tourné pervers ou un accro aux grands combats plus tourné psychologique est non seulement 'orienté' sur ce qui est pervers ou sur le psychologique mais est en plus attiré par ces domaines. C'est ce mécanisme qui explique aussi pourquoi tourner se combine assez souvent avec des noms référant à des genres musicaux et même quelquefois avec des préférences politiques.
} 
idéologie : virer Mac/Pepsi, virer langue, virer scooter, ce qui semble peu probable pour tourner (?? J'ai tourné Mac) ${ }^{31,32}$.

\section{BILAN}

Même si les emplois attributifs de virer et tourner restent nettement moins fréquents que leurs emplois directionnels abstraits introduits notamment par la préposition à, force est de reconnaitre que l'inventaire des verbes attributifs en français s'est encore enrichi de deux nouveaux verbes attributifs. Tourner et virer admettent deux constructions directes, l'une étant attributive à proprement parler, l'autre encore directionnelle et caractérisée par certaines caractéristiques formelles spécifiques, qui la distinguent clairement des constructions attributives. En plus, virer entre également dans une série de locutions attributives dans lesquelles il marque un état transitoire ('être provisoirement') : virer en tête / invaincu.

Dans leurs emplois proprement attributifs marquant un changement d'état, les deux verbes présentent un profil assez ouvert, avec toutefois quelques 'îlots' de lexèmes étroitement liés à des emplois figés, notamment du côté de tourner. Ils ont une orientation nettement péjorative, comme le montrent notamment le sémantisme des principales classes sémantiques des 100 collexèmes les plus attirés par ces verbes et le choix de termes vulgaires pour caractériser les convictions idéologiques et les orientations sexuelles considérées comme déviantes par rapport à la norme sociale. Si le recouvrement lexical et sémantique entre les deux verbes est considérable, l'analyse collexémique distinctive a tout de même permis d'identifier (et d'objectiver) une certaine spécialisation (cf. la section 4.2). Cette spécialisation a été mise en rapport avec certaines nuances de la sémantique de base du verbe lexical : le changement de couleur (virer), la dégénérescence (tourner), le mouvement giratoire qui rend fou (tourner) et le changement de direction bipolaire opportuniste (virer). Dans ce sens, on peut parler de lexical persistence (Hopper 1991) : l'emploi grammaticalisé (le verbe attributif) continue à présenter des restrictions sémantiques qui remontent à l'emploi lexical du verbe, des restrictions qui sont souvent de nature plus abstraite, résultant d'une sorte de transposition.

Arrivé au terme de cette étude, tout n'a certainement pas pu être dit. S'il est clair que l'histoire des constructions attributives est étroitement liée à l'existence de constructions indirectes en à (comme exemplifiées dans la section 5; v. Van Wettere et Lauwers 2017), il reste à examiner les rapports complexes entre la construction

\footnotetext{
${ }^{31}$ Un lecteur remarque que je suis tourné Mac est nettement meilleur.

${ }^{32}$ Notons une analogie frappante entre virer et un emploi attributif assez spécifique du verbe anglais to go : Rolls Royce vire champêtre en proposant un nécessaire à pique-nique ( goes countryside). Voir aussi virer solaire (going solar), virer hispanique (going Spanish), comme Going Romance, le nom des colloques des romanistes) etc. À ne pas confondre avec un autre emploi attributif avec des adjectifs qualificatifs ( to go mad 'devenir fou'), qui se traduit parfois également par virer comme le montre la traduction du titre de l'ouvrage Going Rogue de Sarah Palin : Virer rebelle.
} 
attributive, la construction directionnelle abstraite et la construction indirecte en $\grave{a}$ en synchronie. En outre, notre étude devrait être affinée par une étude de la variation géographique, étude qui dans l'état actuel de la science ne peut pas être entamée à défaut de corpus stratifiés suffisamment vastes.

\section{BIBLIOGRAPHIE}

Audring, Jenny et Geert Booij. 2016. Cooperation and coercion. Linguistics 54(4) : 617-637. Bybee, Joan et David Eddington. 2006. A usage-based approach to Spanish verbs of 'becoming'. Language 82(2) : 323-355.

Damourette, Jacques et Edouard Pichon. 1930-1949. Des mots à la pensée. Essai de grammaire de la langue française. 1911-1940. Paris : D’Artrey. (7 volumes)

Desagulier, Guillaume. 2015. Le statut de la fréquence dans les grammaires de constructions : simple comme bonjour? Langages 197 : 99-128.

Ellis, Nick. 2006. Language acquisition as rational contingency learning. Applied Linguistics 27(1) : $1-24$.

Firth, John R. 1957. Papers in Linguistics, 1934-1951. London : Oxford University Press.

Grevisse, Maurice et André Goosse. 2007. Le Bon Usage. Grammaire française. $14^{\mathrm{e}}$ edition. Louvain-la-Neuve : De Boeck/Duculot.

Gries, Stefan Th. 2014. Coll.analysis 3.5. A script for R to compute perform collostructional analyses. $\quad<\mathrm{http} / / / \mathrm{www}$.linguistics.ucsb.edu/faculty/stgries/teaching/groningen/index. $\mathrm{html}>$

Gries, Stefan Th. et Anatol Stefanowitsch. 2004. Extending collostructional analysis: A corpus-based perspective on 'alternations'. International Journal of Corpus Linguistics 9(1) : 97-129.

Hengeveld, Kees. 1992. Non-verbal predication: Theory, typology, diachrony. Berlin : Mouton de Gruyter.

Heylen, Kris, Thomas Wielfaert, Dirk Speelman et Dirk Geeraerts. 2015. Monitoring polysemy: Word space models as a tool for large-scale lexical semantic analysis. Lingua $157: 153-172$.

Hopper, Paul. 1991. On some principles of grammaticalization. Dans Approaches to Grammaticalization, sous la direction de Elizabeth, Traugott et Bernd Heine, 17-35. Amsterdam : Benjamins.

Kilgarriff, Adam, Vít Baisa, Jan Bušta, Miloš Jakubíček, Vojtěch Kovář, Jan Michelfeit, Pavel Rychlý et Vít Suchomel. 2014. The Sketch Engine: Ten years on. Lexicography 1(1) : 7-36.

Lamiroy, Béatrice et Ludo Melis. 2005. Les copules ressemblent-elles aux auxiliaires? Dans Les périphrases verbales, sous la direction de Hava Bat-Zeev Shyldkrot et Nicole Le Querler, 145-170. Amsterdam : Benjamins.

Lauwers, Peter. 2004. La description du français entre la tradition grammaticale et la modernité linguistique. Étude historiographique et épistémologique de la grammaire française entre 1907 et 1948. Leuven : Peeters.

Lauwers, Peter. 2008. The nominalization of adjectives in French : From morphological conversion to categorial mismatch. Folia Linguistica 42(1-2) : 135-176.

Lauwers, Peter. 2011. The modification of predicative bare nouns in French: A functional analysis. Transactions of the Philological Society 109(1) : 12-40.

Lauwers, Peter. 2014. Copular constructions and adjectival uses of bare nouns in French: A case of syntactic recategorization?. Word $60: 89-122$. 
Lauwers, Peter et Els Tobback. 2010. Les verbes attributifs : inventaire(s) et statut(s). Langages 179-180 : 79-113.

Leeman, Danielle. 1996. Attributs du sujet et verbes attributifs. Linx 34/35 : 187-196.

Leeman, Danielle. 2004. Approche d'une description du sens du verbe tourner (emplois intransitifs). Linx $50: 33-52$.

Melis, Ludo. 2007. La suite " préposition adjectif » et la définition syntaxique de la préposition. Dans Variation et stabilité du français. Des notions aux opérations, sous la direction de Pierre Larrivée, 221-234. Paris : Peeters.

Nowakowska, Malgorzata. 2004. Les adjectifs de relation employés attributivement. Kraków : Wydawnictwo Naukowe Akademii Pedagogicznej.

Nunberg, Geoffrey, Ivan A. Sag et Thomas Wasow. 1994. Idioms. Language 70(3) : 491-538.

Perek, Florent. 2016. Using distributional semantics to study syntactic productivity in diachrony : A case study. Linguistics 54(1) : 149-188.

Stefanowitsch, Anatol. 2013. Collostructional analysis. Dans The Oxford Handbook of Construction Grammar, sous la direction de Thomas Hoffmann et Graeme Trousdale, 290-306. Oxford : Oxford University Press.

Stefanowitsch, Anatol et Stefan Th Gries. 2003. Collostructions : Investigating the interaction of words and constructions. International Journal of Corpus Linguistics 8(2) : 209-243.

Sinclair, John. 1987. Looking up. London : Collins.

Tobback, Els et Peter Lauwers. 2012. Une analyse en miroir de deux verbes évidentiels : s'avérer vs se révéler. Revue Romane 47(1) : 49-75.

Van Wettere, Niek et Peter Lauwers. 2017. La micro-constructionnalisation en tandem : la copularisation de tourner/virer. Langue française 194(2) : 85-104.

Wilmet, Marc. 2007. Grammaire critique du français. $4^{\mathrm{e}}$ édition. Paris : Duculot.

Zeschel, Arne. 2012. Incipient Productivity : A Construction-based Approach to Linguistic Creativity. Berlin : De Gruyter Mouton.

\section{OUVRAGES LEXICOGRAPHIQUES ET SITOGRAPHIE}

Grand Larousse de la Langue française en sept volumes. 1971-1978. Paris : Librairie Larousse.

Le Grand Robert de la langue française [en ligne, 2016] <http://gr.bvdep.com/>

Le Petit Robert : dictionnaire alphabétique et analogique de la langue française. 2010. Paris : Dictionnaires Le Robert.

Le Trésor de la Langue Française informatisé (= TLFi) <http://atilf.atilf.fr/>

Sketch Engine, French Ten Ten Corpus <https://www.sketchengine.co.uk/> 